\title{
Global retrieval of bidirectional reflectance and albedo over land from EOS MODIS and MISR data: Theory and algorithm
}

\author{
W. Wanner, ${ }^{1}$ A. H. Strahler, ${ }^{1}$ B. Hu, ${ }^{1}$ P. Lewis, ${ }^{2}$ J.-P. Muller, ${ }^{3}$ X. Li, \\ C. L. Barker Schaaf, ${ }^{1}$ and M. J. Barnsley ${ }^{4}$
}

\begin{abstract}
This paper describes the theory and the algorithm to be used in producing a global bidirectional reflectance distribution function (BRDF) and albedo product from data to be acquired by the moderate resolution imaging spectroradiometer (MODIS) and the multiangle imaging spectroradiometer (MISR), both to be launched in 1998 on the AM-1 satellite platform as part of NASA's Earth Observing System (EOS). The product will be derived using the kernel-driven semiempirical Ambrals BRDF model, utilizing five variants of kernel functions characterizing isotropic, volume and surface scattering. The BRDF and the albedo of each pixel of the land surface will be modeled at a spatial resolution of $1 \mathrm{~km}$ and once every 16 days in seven spectral bands spanning the visible and the near infrared. The BRDF parameters retrieved and recorded in the MODIS BRDF/albedo product will be intrinsic surface properties decoupled from the prevailing atmospheric state and hence suited for a wide range of applications requiring characterization of the directional anisotropy of Earth surface reflectance. A set of quality control flags accompanies the product. An initial validation of the Ambrals model is demonstrated using a variety of field-measured data sets for several different land cover types.
\end{abstract}

\section{Introduction}

This paper reports on the status of the science and algorithms to be employed in the routine production of a global $1-\mathrm{km}$ land surface bidirectional reflectance distribution function (BRDF) and albedo data product to be generated beginning in mid-1998. This product will be derived from the combined data of the moderate resolution imaging spectroradiometer (MODIS) [Running et al., 1994] and the multiangle imaging spectroradiometer (MISR) [Diner et al., 1991], two key sensors of NASA's Earth Observing System (EOS) on the AM-1 platform. Produced through the MODIS project, it will be available for use in atmospheric correction problems, Earth radiation budget studies, and climate and climate change investigations. It will also allow some inference of land surface properties for use in global land cover classification and biophysical databases for vegetation modeling. Furthermore, it will be employed in the angular correction of MODIS and similar imagery.

Section 2 of this paper gives an overview of BRDF applications, with an emphasis on the coupling between the

\footnotetext{
${ }^{1}$ Department of Geography and Center for Remote Sensing, Boston University, Boston, Massachusetts.

${ }^{2}$ Remote Sensing Unit, University College London, London.

${ }^{3}$ Department of Photogrammetry and Surveying, University College London, London.

${ }^{4}$ Department of Geography, University of Wales, Swansea.
}

Copyright 1997 by the American Geophysical Union.

Paper number 96JD03295.

0148-0227/97/96JD-03295\$09.00 atmosphere and the surface reflectance. In section 3 the MODIS BRDF/albedo product will be discussed with respect to sensor characteristics and the Ambrals BRDF models to be used. Section 4 introduces the algorithm that was developed for the processing, explains the processing philosophy, and describes the product produced. Section 5 offers an outline of current model and planned product validation, and discusses the possible relationship of the BRDF model kernels used to land cover type. The paper closes with conclusions.

\section{Multiangle Effects in Remote Sensing}

\subsection{Bidirectional Reflectance}

The observation that the reflectance of most natural surfaces is anisotropic in nature is relevant to remote sensing because under such circumstances the reflectance of a surface depends both on the viewing and on the illumination angle. With natural scenes this directional anisotropy is caused by the way in which shadows cast by an ensemble of objects such as plants, buildings or topographic slopes are hidden or emerge as a function of the observation angle, and by the intrinsic directionality of material in the scene, for example the leaves of the plants. Mathematically, it is described by the BRDF [Nicodemus et al., 1977].

Variations of view angle across images and between images occur naturally due to a wide swath width (as for the advanced very high resolution radiometer (AVHRR) or MODIS) or to along-track off-nadir viewing capabilities (as for the along-track scanning radiometer (ATSR-2) or MISR). Variations in the solar angle are caused by variations in the time of day, season and latitude of observations and are also 
determined by the orbital characteristics of the satellite. Unless corrections for the BRDF are made, comparisons of surface reflectance observations across images from such instruments or between them are difficult or impossible. A simple example is given by the problem of mosaicking together AVHRR data from different orbits to obtain a composite image, where the orbital border will be visible due to the difference in the Sun-view geometry in the two or several parts (see $\mathrm{Li}$ et al. [1996] for an illustration).

The need to take BRDF effects into account when conducting land surface studies, for example, land cover classification, has increasingly been recognized [e.g., Wu et al., 1995; Cihlar et al., 1994; Gutman, 1994; Moody and Strahler, 1994]. It is known that current vegetation indices retain some angular effects due to the differences in the BRDF of different wave bands [e.g., Wu et al., 1995]. It may even be desirable to standardize observations to angles where no observations were acquired, for example, to nadir view and/or Sun angle [Leroy and Roujean, 1994].

The BRDF may also be used to derive albedos, which are mathematically defined as weighted integrals (averages) of the BRDF. Surface albedos play an important role in global and regional climate since they determine the surface energy balance, for example, the heat fluxes linked to evapotranspiration [Kustas et al., 1989]. The BRDF and its integrals are also important for atmospheric correction in remote sensing [Kaufman, 1989], where errors of up to $10 \%$ or more may be made when BRDF effects are disregarded. We will discuss albedos and atmospheric correction in more detail farther on. Recently, there have also been indications that knowledge of the BRDF function can be valuable in cloud detection [d'Entremont et al., 1996; see also DiGirolamo and Davies, 1994], opening up the possibility of improved cloud detection in the absence of thermal data.

Finally, the BRDF can be interpreted to infer land surface properties. Since the angular dependence of the reflectance is driven by the optical properties of the scattering medium and by its three-dimensional structure, one may attempt to retrieve the dominant parameters describing these properties from the observed BRDF functions.

Initially, such retrievals will be experimental, and the main focus of BRDF/albedo work will be on semiempirical descriptors of the BRDF that can be used for angle corrections and albedo calculations without requiring detailed physical modeling of the scene viewed. This is the path taken by the MODIS BRDF/albedo product. As more experience is accumulated, however, and as more computer power becomes available, more elaborate retrievals of physical parameters may be attempted [Goel, 1989; Pinty and Verstraete, 1991]. The MODIS land cover product [Strahler et al., 1996a] will make use of reflectances corrected for angular effects but will also take into consideration the observed BRDF shape itself.

\subsection{Surface BRDF-Atmosphere Coupling}

Atmospheric correction algorithms for operational processing are commonly based on expressing the total transmittance of radiation from the top of the atmosphere to the ground as the sum of a direct and a diffuse component (see Kaufman [1989] for a general treatment of atmospheric cor- rection in remote sensing). One then arrives at expressions such as that suggested by Li [cf. Strahler et al., 1995a], which is similar to the formulation to be used for MODIS atmospheric correction at visible and near-infrared wavelengths [see Vermote et al., 1995, this issue]. We have

$$
\rho_{\text {toa }}=\rho_{\text {path }}+\frac{\mathbf{t}\left(\mu_{s}\right) \mathbf{R t}\left(\mu_{v}\right)-e^{-\tau / \mu_{s}}|\mathbf{R}| e^{-\tau / \mu_{v}}}{1-\overline{\overline{\rho_{s}}} \rho_{a}},
$$

where $\mu_{s}$ is the cosine of the solar zenith angle, $\mu_{v}$ that of the view zenith angle, $\rho_{\text {toa }}$ the reflectance at the top of the atmosphere, $\rho_{\text {path }}$ the reflectance due to path radiance, $\rho_{a}$ the downward hemispherical reflectance of the atmosphere for isotropic light entering at its base, $\rho_{s}\left(\mu_{s}, \mu_{v}, \phi\right)$ the directional surface reflectance (surface BRDF), with $\phi$ the relative azimuth between Sun and view zenith $\left(\theta_{s}\right.$ and $\left.\theta_{v}\right)$, and $\tau$ the optical thickness; the vector $t$ and matrix $R$ are given by

$$
\begin{aligned}
\mathbf{t}(\mu) & =\left[e^{-\tau / \mu}, t_{d}(\mu)\right], \\
\mathbf{R} & =\left[\begin{array}{cc}
\rho_{s} & \rho_{b}\left(\mu_{s}\right) \\
\overline{\rho_{s}}\left(\mu_{s} ; \mu_{v}, \phi\right) & \overline{\overline{\rho_{s}}}
\end{array}\right] .
\end{aligned}
$$

In these expressions, $t_{d}$ is the diffuse transmittance of the atmosphere, $\rho_{b}$ is the directional-hemispherical integral ("black-sky albedo") of the BRDF,

$$
\rho_{b}\left(\mu_{s}\right)=\frac{1}{\pi} \int_{0}^{2 \pi} \int_{0}^{\pi / 2} \rho_{s} \cos \left(\theta_{v}\right) \sin \left(\theta_{v}\right) d \theta_{v} d \phi,
$$

$\overline{\rho_{s}}\left(\mu_{s} ; \mu_{v}, \phi\right)$ is the diffuse irradiance that is directly scattered into the viewing direction $\left(\mu_{v}, \phi\right)$ under illumination from $\mu_{s}$,

$$
\begin{aligned}
& \overline{\rho_{s}}\left(\mu_{s} ; \mu_{v}, \phi\right)= \\
& \frac{\int_{0}^{2 \pi} L_{l}\left(\mu_{s} ; \mu, \phi^{\prime}\right) \rho_{s}\left(\mu, \mu_{v}, \phi^{\prime}-\phi\right) \cos (\theta) d \theta d \phi^{\prime}}{\int_{0}^{2 \pi} L_{\downarrow}\left(\mu_{s} ; \mu, \phi^{\prime}\right) \cos (\theta) d \theta d \phi^{\prime}}
\end{aligned}
$$

with $L_{\downarrow}\left(\mu_{s} ; \mu, \phi^{\prime}\right)$ the downwelling diffuse irradiance distribution with the Sun at $\mu_{s}$, and $\overline{\overline{\rho_{s}}}$ is the diffuse-to-diffuse reflectance under illumination from $\mu_{s}$,

$$
\overline{\overline{\rho_{s}}}=\frac{1}{\pi} \int_{0}^{\pi / 2} \overline{\rho_{s}}\left(\mu_{s} ; \mu, \phi\right) \cos (\theta) \sin (\theta) d \theta d \phi .
$$

Note that $\mathrm{t}\left(\mu_{s}\right)$ and $\mathrm{t}\left(\mu_{v}\right)$ contain only atmospheric properties, namely, the direct and diffuse parts of the transmittance, while $\mathbf{R}$ contains only surface reflectance properties, although partly under the prevailing conditions of diffuse illumination, which depend on the atmospheric condition. The determinant $|\mathbf{R}|$ has the physical meaning of a correction term for using $\rho_{s}$ in multiple bouncing between surface and atmosphere.

These equations clearly demonstrate the coupling between surface reflectance properties and atmospheric radiative transport. Given the atmospheric parameters, the BRDF $\rho_{s}$ can be retrieved only if the integral reflectance expressions occurring are known, which in turn depend on the BRDF and the atmospheric state.

Approximations that can be made are to simplify the treatment of multiple scattering to be mainly based on the terms 
involving $t_{d}\left(\mu_{s}\right) t_{d}\left(\mu_{v}\right)$, or to approximate $\overline{\rho_{s}}$ by $\rho_{b}$. However, the error made can be significant under turbid conditions [Lewis and Barnsley, 1994]. Approximating $\overline{\overline{\rho_{s}}}$ by the bihemispherical integral of the BRDF (the "white-sky albedo" $\rho_{w}$, which is the normalized integral of $\rho_{b}\left(\theta_{s}\right) \cos \left(\theta_{s}\right)$ over the Sun zenith) is less problematic, since the multibounce radiation between surface and atmosphere will tend to an isotropic distribution.

If a simultaneous, iterative retrieval of surface reflectance and the coupling integrals is not possible, approximate values will have to be used for the BRDF integrals. In practice, these may be taken from previous retrievals or estimated from the surface reflectance and ancillary data. In the past, the most commonly used assumption has been that the surface is Lambertian, which breaks the coupling but introduces a potentially important error, as is demonstrated in the next section.

\subsection{Magnitude of BRDF Effects in Atmospheric Correction}

Work by Lee and Kaufman [1986] showed that, even in nonturbid conditions, the assumption of a Lambertian surface leads to large errors in predicted upward radiance in the backscattering portion of the hemisphere (the situation is much more favorable in the forescattering direction), especially for large solar zenith angles (e.g., 60 $)$. This study was conducted for a savanna data set [Kriebel, 1978]. In a more recent study, Vermote et al. [1995, this issue] performed atmospheric corrections of reflectance data that were obtained from a Hapke model [Pinty et al., 1989] fitted to directional reflectances of a plowed field with the Sun at $30^{\circ}$ zenith angle [Kimes, 1985]. Corrections were carried out first using the assumption of a Lambertian surface, then repeated using the BRDF reconstructed from that "Lambertian" retrieval. In this manner a one-step iteration was carried out. The respective solutions were compared for three different optical depths. For a nonturbid atmosphere, $\tau=0.01$, the Lambertian assumption does not lead to serious errors. For $\tau=0.23$ the relative error is already about $10-15 \%$ at view zenith angles of $\pm 60^{\circ}$ and less at smaller angles. The error of the solution after one iteration is reduced to about 3-4\%. In a turbid situation, $\tau=0.5$, the error made with the Lambertian assumption is large, about $30 \%$ at $+60^{\circ}$ (forescattering) and $12 \%$ at $-60^{\circ}$, with smaller errors at smaller view zenith angles. After the first iteration, this error is reduced to 20 and
$5 \%$, respectively. A second iteration, based on the BRDF from the first iteration, reduces the error to 14 and $1 \%$, respectively.

We have conducted a similar study using a Ross-thick/Lisparse kernel-driven BRDF model [Wanner et al., 1995a]. Three different types of BRDF were investigated: strong volume scattering (a croplike canopy), strong geometric-optical scattering (a sparse woodland canopy), and an intermediate case. The atmospheric model used was a U.S. Standard Atmosphere and continental aerosol, and the $6 \mathrm{~S}$ atmospheric correction code was used [Vermote et al., 1997]. Table 1 shows the ranges of RMS error, in percent, that were observed for these cases for different optical depths, and when using a Lambertian assumption for atmospheric correction and when not, performing a one-step iteration. Results are given for the red and near-infrared wave bands and for the surface BRDF and the bihemispherical integral of the BRDF (the white-sky albedo). In both cases the errors which arise clearly increase with optical depth but are noticeably smaller in the non-Lambertian case when compared to that using the Lambertian assumption. Employing an isotropic surface reflectance in atmospheric correction, errors in the surface reflectance between about 2 and $16 \%$ are made. A one-loop correction, however, reduces the errors to a range from 1 to $3 \%$ in all but the most turbid case, where the errors are also reduced considerably. The situation is similar with the bihemispherical reflectance. The error made is reduced by a significant factor if a one-step iteration loop is performed.

These results show clearly that errors of up to $10 \%$, and at some angles and under turbid conditions more, occur if BRDF effects are not taken into account in atmospheric correction. The difference between the first and the second iteration in the turbid case of the study of Vermote at al. [1995] illustrates that even using a BRDF that is only approximately correct can be improved upon noticeably by using a BRDF that is closer to the real anisotropy. The conclusion from all the studies is that it is worthwhile, even necessary, to use BRDF corrections in atmospheric correction, unless the conditions are not very turbid. It also is clear that the size of the error that remains depends on the accuracy of the BRDF used. However, an iteration in one or two steps leads to sufficiently accurate results and should therefore always be carried out in operational atmospheric correction where BRDF information is available. Given the central importance of surface reflectance to most of the goals

Table 1. Percent RMS Error Ranges in BRDF and Bihemispherical Albedo Retrieval Using Lambertian and Non-Lambertian Assumptions in Atmospheric Correction and Different Optical Depths $\tau$

\begin{tabular}{|c|c|c|c|c|c|c|c|}
\hline & \multirow[b]{2}{*}{ Band } & \multicolumn{2}{|c|}{$\tau=0.15$} & \multicolumn{2}{|c|}{$\tau=0.30$} & \multicolumn{2}{|c|}{$\tau=0.50$} \\
\hline & & Lambertian & Non-Lamb. & Lambertian & Non-Lamb. & Lambertian & Non-Lamb. \\
\hline BRDF & $\begin{array}{l}\text { red } \\
\text { NIR }\end{array}$ & $\begin{array}{l}2.3-5.2 \\
1.5-5.7\end{array}$ & $\begin{array}{l}0.4-1.3 \\
0.1-0.7\end{array}$ & $\begin{array}{c}5.9-10.6 \\
2.7-7.7\end{array}$ & $\begin{array}{l}0.7-2.9 \\
0.4-2.5\end{array}$ & $\begin{array}{l}7.4-16.2 \\
4.1-14.2\end{array}$ & $\begin{array}{l}1.6-5.9 \\
1.2-6.2\end{array}$ \\
\hline $\begin{array}{l}\text { Bihem. } \\
\text { Albedo }\end{array}$ & $\begin{array}{l}\text { red } \\
\text { NIR }\end{array}$ & $\begin{array}{l}1.8-7.1 \\
1.0-1.9\end{array}$ & $\begin{array}{l}0.2-1.4 \\
0.2-0.8\end{array}$ & $\begin{array}{l}0.8-9.5 \\
1.0-3.0\end{array}$ & $\begin{array}{l}0.3-1.7 \\
0.3-0.6\end{array}$ & $\begin{array}{c}1.8-14.6 \\
0.5-5.1\end{array}$ & $\begin{array}{l}0.3-3.6 \\
0.5-1.5\end{array}$ \\
\hline
\end{tabular}

The ranges given correspond to three different shapes of the bidirectional reflectance distribution function (BRDF): one that is dominated by volume scattering, one that is dominated by surface scattering, and one that is a mix of the two. 
to be achieved in optical remote sensing, this implies that deriving a BRDF/albedo product is key to obtaining accurate results from remotely sensed optical data.

This brief review has focused on the specific case of the atmospheric correction problem. However, by implication, similar effects are to be expected in principle for radiation budget studies and the radiation treatment in climate and weather models. Clearly, the anisotropic nature of the reflectance of the Earth's surface plays a role in shaping the energy flux in the atmosphere close to the surface and needs to be taken into consideration in any surface data retrieval or radiative tranfer modeling.

\subsection{Albedo}

Integrals of the BRDF play a role beyond that of atmospheric correction. Accurate global albedo values are required for global and regional climate and climate change studies. Zhang et al. [1995] point out that over land the average shortwave reflectivity of clouds is only 1.5 times higher than that of the surface, making surface albedo an important influence. These authors go on to state that current uncertainty in land surface albedo is more important than current uncertainty in cloud albedo with respect to determining net surface shortwave fluxes. With respect to climate models, Henderson-Sellers and Wilson [1983] state that an absolute accuracy of $5 \%$ is required for a global albedo data set. The albedo values used by different climate models for identical regions still differ by several percent (see $\mathrm{Li}$ and Garand [1994] for an overview). Gutman [1994] addresses the problem that albedo over dark targets, like rain forests, is more problematic than over land covers with higher reflectivity, and that errors here can easily be $20 \%$. Sellers [1993] cites a need for a $2 \%$ absolute accuracy for global albedo in climate models. Meeting this need for accurate albedo data is a prime objective for remote sensing, one that has not been fully met up to date.

In attempts to bridge this gap, global albedo has been derived using a number of techniques utilizing radiation flux and budget assessments but hitherto not from bidirectional reflectance distribution functions. Zhang et al. [1995] assume Lambertian reflectance in their study of surface and top-ofatmosphere radiative fluxes. The albedos used are based on a few land cover classes and wind speeds over oceans and are given as a function of solar zenith angle and season. Dropping the Lambertian assumption will be the obvious next step in the development. A method also discussed by Zhang et al. [1995] is to use albedos derived from the Earth radiation budget experiment (ERBE) to fit measurements at hand, depending on land cover type. Li and Garand [1994] investigate deriving surface albedo from top-of-atmosphere albedos in a clear sky situation and find an accuracy of a few percent. They point out that bidirectional effects have not been given proper attention even though they are among the decisive factors in determining surface albedo from satellite measurements. These authors also use a Lambertian albedo for the land surface. The albedos of the International Satellite Land Surface Climatology Project (ISLSCP) data sets [Sellers et al., 1994] are derived using a two-stream approximation. Problems with directional effects in AVHRR data in general and their influence on surface albedo estimates are discussed by Gutman [1994] and Cihlar et al. [1994].
As a consequence, current methods for estimating albedo from land cover properties or radiation flux budgets will be replaced by albedo products derived from directional reflectance measurements utilizing BRDF models of the land surface, such as the MODIS BRDF/albedo product. These new products will also have the advantage of having much finer spatial resolution than that of current data $\left(2.5^{\circ} \times 2.5^{\circ}\right.$ for ERBE; $1^{\circ} \times 1^{\circ}$ for the ISLSCP database). This will allow not only a closer correlation of albedos with land cover classes, which is needed for parameterizations in the land surface schemes of climate models, but also quantifications of subgrid variability and derivation of better coarse-scale averages.

\section{MODIS BRDF/Albedo Product}

\subsection{Product Outline}

The MODIS BRDF/albedo product will provide the full surface BRDF as well as black-sky (directional) and whitesky (diffuse) albedos, these being intrinsic surface albedos. It will be produced globally (over land surfaces only) from a combination of MODIS and MISR data, at a spatial resolution of $1 \mathrm{~km}$. BDRF and albedos will be provided in seven spectral bands ranging from 0.47 to $2.13 \mu \mathrm{m}$ [see Running et $a l ., 1994]$ and in three broadbands ranging from 0.3 to $0.7 \mu \mathrm{m}$, from 0.7 to $3.0 \mu \mathrm{m}$, and from 0.3 to $3.0 \mu \mathrm{m}$. The product will be produced once every 16 days (the EOS AM-1 platform and MISR 2-look repeat rate) and will be based on the semiempirical Ambrals BRDF model [Wanner et al., 1995a; Strahler et al., 1996b; Lewis, 1995]. Results from inverting the empirical modified Walthall BRDF model [Walthall et al., 1985; Nilson and Kuusk, 1989] will also be recorded. A separate quarter-degree resolution product will be provided for global modeling purposes. This product will also carry information on sub-scale variability. Principal Investigators on the MODIS BRDF/albedo product are A. H. Strahler of Boston University and J.-P. Muller of University College London.

\subsection{Angular Sampling From MODIS and MISR}

Two key issues for a BRDF/albedo product are the angular sampling coverage available and the expected rate of loss of angular data due to cloud cover. Angular sampling is given by instrumental and orbital characteristics [Barnsley et al., 1994]. The EOS AM-1 platform carrying both MODIS and MISR will have a polar orbit with a $10^{30}$ morning equatorial crossing time. The PM-1 platform, to be launched in the year 2000 with a second MODIS sensor, will have a $13^{30}$ early afternoon crossing time. Data from this second MODIS will be included in the MODIS BRDF/albedo processing chain when it is available.

The MODIS sensor will image the Earth's surface across track with a swath width of $2330 \mathrm{~km}$ [Running et al., 1994]. The viewing zenith angle will vary between $\pm 55^{\circ}$, or about $\pm 61^{\circ}$ at the surface. The repeat cycle is 2 days at most and less than 1 day at latitudes greater than about $30^{\circ}$. Orbital overlap thus makes it well suited for accumulating multiangle reflectance data over a period of time. Spatial resolution at nadir is $250 \mathrm{~m}$ for the red and near-infrared bands and $500 \mathrm{~m}$ for the other land bands. 

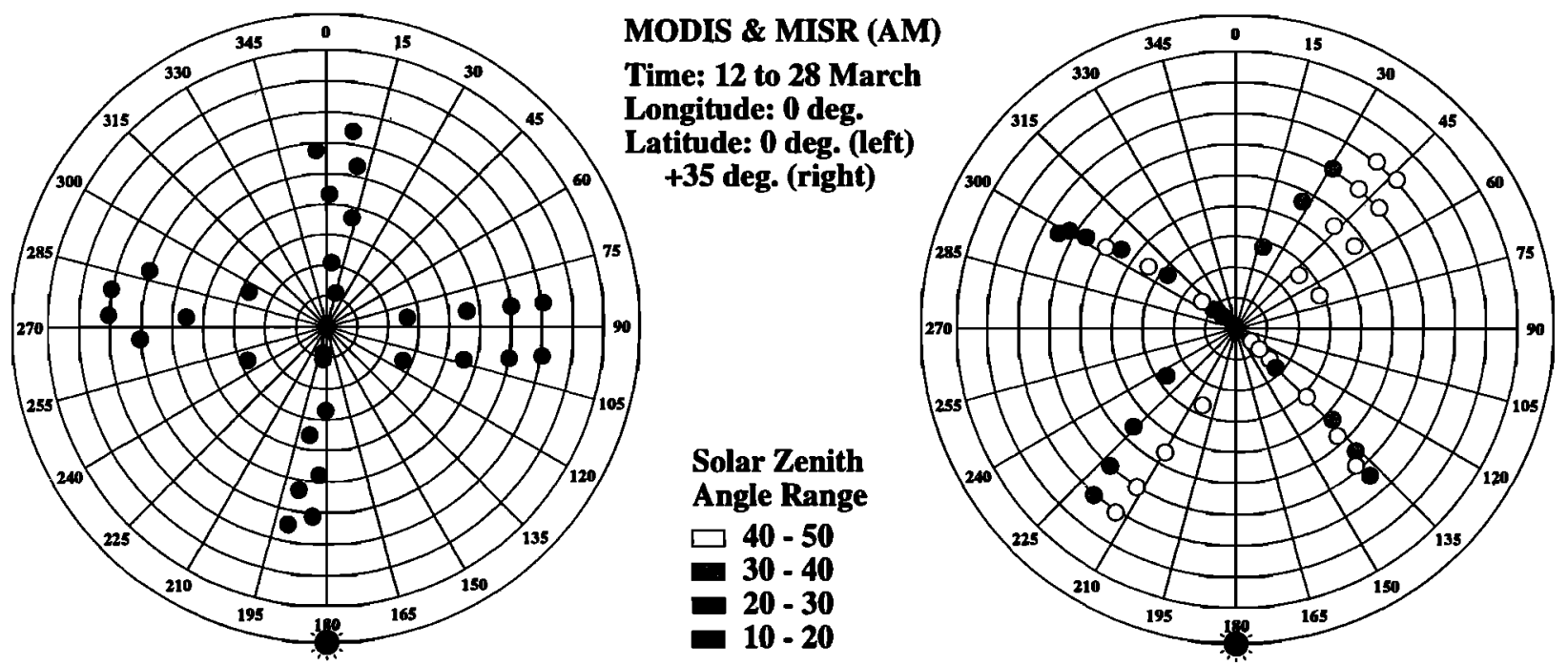

Figure 1. Distribution of observations from moderate resolution imaging spectroradiometer (MODIS) and multi-angle imaging spectroradiometer (MISR) in the viewing hemisphere. The time period is March $12-28$ (16 days). The left plot is for the equator; MODIS observations are close to the principal plane; MISR observations are on the cross-principal plane. The right plot is for a latitude of $35^{\circ}$ north; both MODIS and MISR are sampling away from the principal plane, with MISR a little closer to it. Farther north, MISR is closer to the principal plane. The solar zenith angle range in both cases is about $15^{\circ}$. Data and plots produced by the Xsatview software (M. Barnsley and K. Morris, University College London) from approximate representations of the satellite orbits.

MISR will image along track using nine separate push broom cameras [Diner et al., 1991]. Four of these are aft looking, four fore pointing, and one is nadir viewing. Observations will be made in four spectral bands at visible and near-infrared wavelengths that are similar to those of MODIS. The potential spatial resolution of MISR observations is $275 \mathrm{~m}$, but data will be made available at a standard spatial resolution of $1.1 \mathrm{~km}$. MISR's swath width is $364 \mathrm{~km}$, allowing for a 9-day global repeat cycle, with the two-repeat cycle being 16 (not 18 ) days at the equator.

Angular sampling sufficient for BRDF model inversions will be achieved by combining data from MODIS and MISR in the four corresponding spectral bands and by accumulating data over a period of 16 days. In the absence of clouds this leads to between 30 multiangular observations available at the equator and about 55 observations at $60^{\circ}$ latitude, subject to variation with season. Using a coarse statistic of mean global cloud cover probability [Wylie and Menzel, 1989; Wylie et al., 1994] as a function of latitude, one may predict that generally, about 15 to 20 multiangular observations will be available at all latitudes except at the equator, where abundance of cloudy conditions is likely to create problems.

With this number of observations available, on the average, sufficient angular coverage for a BRDF model inversion is to be expected in most cases. Since MODIS scans across track and MISR along track, the respective views will generally represent different parts of the BRDF [Barnsley et al., 1994]. When one sensor views the cross-principal plane of the BRDF, the other views the principal plane, and vice versa. Figure 1 illustrates this, showing two typical cases for a particular latitude and time of year. With two sweeps across it, sampling of the viewing hemisphere is good in all cases. Sampling of the illumination hemisphere, however, is limited. Any one string of MISR data is acquired for only one solar zenith angle, while the MODIS observations display a small but valuable variation in that angle during the sampling period.

\subsection{Kernel-Based Semiempirical BRDF Models}

The MODIS BRDF/albedo product will be based on a semiempirical, kernel-based modeling approach introduced by Roujean et al. [1992] for top-down BRDF modeling. Mathematically, this type of model has the following form (here using two kernels and a constant):

$$
R\left(\theta_{i}, \theta_{v}, \phi\right)=f_{i}+f_{v} k_{v}\left(\theta_{i}, \theta_{v}, \phi\right)+f_{s} k_{s}\left(\theta_{i}, \theta_{v}, \phi\right),
$$

where $R$ is the reflectance, $k_{v}$ and $k_{s}$ are kernel functions, and $f$ are the weights of these functions (the model parameters). The kernels depend only on the viewing and illumination geometry. They describe basic BRDF shapes into which the full BRDF may be decomposed.

In this kernel-based BRDF model, the BRDF is given as a linear superposition of kernel shapes, which are chosen to represent different types of scattering with typical influences on the BRDF. In the above formulation, for example, $k_{v}$ stands for volume scattering as described by radiative transfer theories, and $k_{s}$ for surface scattering as derived from geometric-optical theories. The kernel that goes with the constant $f_{i}$ is the isotropic Lambertian kernel, unity. Although the kernel functions used can be empirical (as in the Walthall et al. [1985] model), they are best derived from a physical theory through approximation, making the resulting model semiempirical. Mathematically, the weights $f$ may be expressed in terms of physical properties of a scene, 
such as leaf area index, shape and height of trees, surface reflectance, etc. Through inversion, however, only the three quantities $f$ are retrieved, providing the relative influence of the respective type of scattering on the directional signal observed and allowing a rough characterization of the structural characteristics of the surface observed.

The kernel-based approach to BRDF modeling has been discussed and justified by Roujean et al. [1992] and Wanner et al. [1995a]. Depending on the scene, it may be understood in different ways. In a mixed scene composed of two areas with different BRDFs, for example, a grassland-forest mixed pixel, the superposition represents the respective areal contributions of the mainly shadow-driven (geometric-optical) BRDF of the forest and the turbid medium-type BRDF of the grassland. Adjacency effects are neglected, which is admissible unless the mosaic is very fine or the zenith angle very large. However, even in a homogeneous scene, volume and surface scattering may still be both present. For example, in a forest canopy the geometric scattering is given by the inter crown gaps, whereas the volume scattering is given by the gaps between the leaves. The superposition then describes first- and second-order scattering, while neglecting the coupling of the two. From a practical standpoint it is perhaps most accurate to say that the BRDF observed is decomposed into the two components represented by the two basic types of scattering. Volume and surface scattering produce BRDF functions that are semiorthogonal due to an increase in reflectance with zenith angle in the former and a decrease in the latter case.

Kernel-driven models have been proven successful in application to AVHRR, advanced solid state array spectroradiometer (ASAS), laboratory, and field-measured multiangular reflectance data and have been shown to fit observed BRDF data well [ Roujean et al., 1992; Leroy and Roujean, 1994; Wu et al., 1995; Wanner et al., 1995b; Privette and Vermote, 1995; Strahler et al., 1995b; Li et al., 1996; Barnsley et al., 1997a; Hu et al., 1996; White et al., 1996]. Some examples of the latter will be given in section 5. A kernelbased model is also being used for the BRDF/albedo product of the polarization and directionality of the Earth's radiation (POLDER) project [Deschamps et al., 1994; Leroy et al., this issue].

\subsection{Ambrals BRDF Model}

The kernel-based BRDF model to be used for production of the MODIS BRDF/albedo product was chosen to be the Ambrals BRDF (Algorithm for MODIS bidirectional reflectance anisotropy of the land surface) BRDF model, as defined by the kernels used in it. The rationale for choosing this model is given in section 3.5. The kernels used are based on theory by Roujean et al. [1992], Ross [1981], $L i$ and Strahler [1992], Li [Strahler et al., 1994; Wanner et al., 1995a], and Cox and Munk [1954].

The Ambrals model provides two choices each for the volume scattering and for the surface scattering kernel. The kernels to be used in a particular case depend on the characteristics of the multiangular observations available. The two kernel expressions derived for volume scattering are based on two different approximations to a single-scattering ra-
Principal Plane Values of Kernels
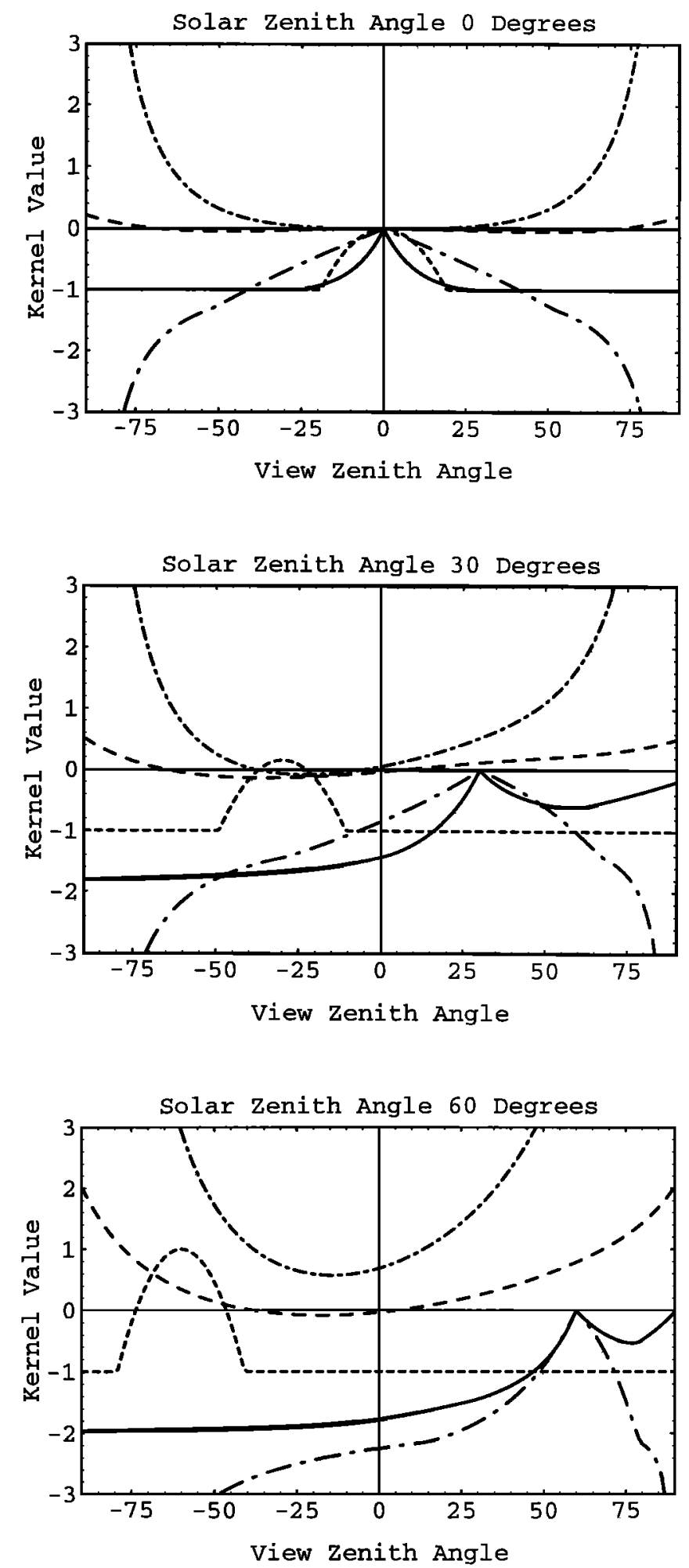

Figure 2. Principal plane values of the kernels for three different solar zenith angles. Dashed-dotted curve, Rossthin; dashed curve, Ross-thick; short-dashed curve, specular; solid curve, Li-dense; long-dashed-dotted curve, Li-sparse. The Li-sparse kernel is calculated for $b / r=1$ and $h / b=2$ (round, low crown), the Li-dense kernel for $b / r=2.5$ and $h / b=2$ (prolate, high crown). 
Table 2. Ambrals Model Kernel Combinations to be Used in Generating the MODIS BRDF/Albedo Product

\begin{tabular}{lllll}
\hline Model & Applicable Scene Type & LAI & $b / r$ & $h / b$ \\
\hline 1, Ross-thin/Li-sparse & $\begin{array}{c}\text { scenes dominated by weak volume scattering } \\
\text { and simultaneous shadow casting } \\
\text { scenes dominated by weak volume scattering } \\
\text { 2, Ross-thin/Li-dense }\end{array}$ & $\begin{array}{c}\text { low or } \\
\text { very high } \\
\text { low or } \\
\text { very high }\end{array}$ & 1.0 & 2.0 \\
3, Ross-thick/Li-sparse & $\begin{array}{c}\text { scenes dominated by volume scattering } \\
\text { and simultaneous shadow casting } \\
\text { scenes dominated by volume scattering } \\
\text { and simultaneous mutual shadowing }\end{array}$ & moderate & 1.0 & 2.0 \\
4, Ross-thick/Li-dense & $\begin{array}{c}\text { moderate } \\
\text { 5, Cox-Munk/Li-sparse }\end{array}$ & 2.5 & 2.0 \\
& $\begin{array}{c}\text { scenes dominated by shadow casting and } \\
\text { simultaneous specular forward scattering }\end{array}$ & N/A & 1.0 & 2.0 \\
\hline
\end{tabular}

MODIS, moderate resolution imaging spectroradiometer. Note that for each kemel combination the weights attributed to the individual kemels can still range from making volume scattering dominant over surface scattering or vice versa. This may be due to the respective areal proportions of the scene components, or to a mix of the two scattering types in a homogeneous scene.

diative transfer theory of Ross [1981], one for large values of the leaf area index ("thick" approximation) [Roujean et al., 1992], and one for small values ("thin" approximation) [Wanner et al., 1995a]. For geometric surface scattering, two types of $\mathrm{Li}$ kernels are available. These were derived from the geometric-optical mutual shadowing BRDF model for forest canopies by Li and Strahler [1992]. The first of these is an approximation for "sparse" spacing of discrete objects (crowns) and the second for "dense" spacing of objects (crowns) [Strahler et al., 1994; Wanner et al., 1995a]. The Li-sparse kernel is mainly driven by the way shadows emerge and are hidden in the scene, while the Li-dense kernel is dominated by the sunlit crowns as seen under conditions of mutual shadowing in both viewing and illumination directions. For MODIS BRDF/albedo processing, the Li-sparse kernel is formulated to represent spheroids that are relatively close to the ground $(b / r=1, h / b=2$, where $b$ is the crown vertical radius, $r$ the crown horizontal radius, and $h$ the height to center of crown). The Li-dense kernel is formulated to represent prolate crowns that are located some distance above the ground $(b / r=2.5, h / b=2)$. For general use of the Ambrals model, other choices of these parameters can be made.

In cases where the forward scattering direction of the BRDF has been sampled, a Cox-Munk kernel has been developed [Strahler et al., 1995a, update] to model contributions from subresolution water bodies, flooded fields, and melting ice. This kernel is based on a theory of Sun glint on the ocean by Cox and Munk [1954]. A wind speed parameter of $5 \mathrm{~m} / \mathrm{s}$ is used.

Figure 2 shows the shape of these five Ambrals model kernels on the principal plane for three different solar zenith angles. Kernels may be normalized before use to have comparable ranges of values or integrals. Note that volume scattering and surface scattering kernels are, to a large degree, linearly independent.

Using these kernels, the Ambrals BRDF model can acquire five distinct forms, which allows good fits to the variety of BRDF shapes expected from global BRDF observations at the kilometer scale. The Ross-thin kernel is combined with either the $\mathrm{Li}$-sparse or the $\mathrm{Li}$-dense kernel, as is the Rossthick kernel. The Cox-Munk kernel is combined with the Li-sparse kernel. These five model variants, also listed in Table 2, will be used to produce the MODIS BRDF/albedo product. Tests have shown that for a variety of land cover types at least one of these models provides a good fit, although for some land cover types, several models may do well due to the fact that they share the kernel that accounts for most of the BRDF shape (see section 5 of this paper and Wanner et al. [1995b] and Hu et al. [1996]).

\subsection{Rationale in Choosing the Ambrals BRDF Model}

Quite a number of BRDF models are available in the literature, ranging from simple empirical to rather complex numerical models that more closely represent the physical features of the plant types being modeled (Strahler [1994] gives an overview). The majority of these were derived from a forward modeling perspective. Geometric and optical properties of vegetation are used to compute the most prominent features of radiation scattering and shadow casting in plant canopies and to derive the BRDF. While being very valuable in providing an insight into the physics of the interaction of light with vegetation, they are not ideally suited for remote sensing applications. Forward modeling represents a bottom-up approach, while remote sensing requires dealing with the inverse problem. The perspective that needs to be at the core of the modeling is top-down.

The rationale for choosing the Ambrals BRDF model for the operational inversions of MODIS BRDF/albedo processing is outlined by four requirements that were met by this model.

1. The model is required to be reflectance-based to eliminate the need to rely on ancillary databases of vegetation properties, soil brightnesses, etc. Global databases of these properties do not exist currently with the required spatial resolution and mostly are of unknown quality. Furthermore, the approach chosen should be flexible enough to model the BRDFs of the major types of vegetation and nonvegetated 
surfaces. This, to some extent, precluded the use of just one single BRDF model for all land cover types.

The Ambrals model meets these requirements in that the inversion of three parameters is achievable from the available multiangular sampling and that no additional parameters need to be predetermined. It has been successfully applied to a number of observed BRDF data sets [Privette and Vermote, 1995; Strahler et al., 1995b; Wanner et al., 1995b; Hu et al., 1996; White et al., 1996]. A similar model is to be used for operational BRDF retrievals or POLDER [Leroy et al., this issue].

2. The model used should be robust in several respects. It should be robust with regard to inversion from limited angular sampling and robust against noise in the input data. It is essential that it should not assume that the pixels viewed possess a homogeneous land cover (an assumption implicit in most BRDF models). Globally, the number of mixed pixels heterogeneous at the 100-m-scale is large. Also the BRDF model needs to be able to model BRDF effects caused by hilly and rugged topography and by rough soil surfaces.

Because of the top-down modeling approach taken in kernel-driven models, discussed in the previous section, the Ambrals BRDF model meets these requirements well. Noise sensitivity is discussed in section 3.7.

3. There should be a mathematically clear way of scaling the model, and hence the derived data product, across a range of spatial scales. The MODIS BRDF/albedo product will be generated at a spatial resolution of $1 \mathrm{~km}$, but the results may be applied at coarser resolutions up to climate-model resolutions of, e.g., $2.5^{\circ}$. Since the BRDF is a nonlinear function of at least three angles, such spatial scaling is not a straightforward operation and neither is the spatial averaging of albedos when taking a varying BRDF effect into account.

The Ambrals model scales spatially due to its linear properties: the parameters of the model at a coarser spatial scale may be expressed through weighting of the parameters of each kernel according to their proportion of area. This will allow degrading the MODIS BRDF/albedo product to any resolution desired for a specific modeling purpose.

4. The model must have a speedy inversion. This precludes the use of numerical inversion techniques, typically required for the inversion of physical and numerical BRDF models [cf. Goel, 1989]. Currently, only a model that has a mostly analytical inversion is a feasible candidate.

The Ambrals model may be inverted analytically by solving the set of linear equations derived from minimizing an appropriate error function through matrix inversion [Lewis, 1995]. The model inverts extremely rapidly for this reason and is consequently ideally suited for large-scale global BRDF inversion.

\subsection{Albedo From MODIS}

The influence of atmospheric state on the radiative fluxes at the surface becomes an issue when deriving albedo. Albedo is defined as the ratio between the hemispherical upwelling and the downwelling fluxes, the latter depending on the amount of diffuse scattering occurring in the atmosphere due to its aerosol and water vapor load. This makes the albedo itself dependent on the atmospheric state at the time of observation. Albedo in this form, while characterizing ac- curately the radiative fluxes at the time of observation, is not directly usable in biospheric and climate modeling, where the atmosphere applied and/or computed may be different from that observed and may vary as a simulation proceeds. What is needed for modeling is an average or hemispherical reflectance in form of an intrinsic surface property that is not dependent on a particular atmosphere and time.

In order to meet this demand, the MODIS BRDF/albedo product will provide two measures of albedo in the form of the directional-hemispherical and bihemispherical integrals of the BRDF, derived through the Ambrals BRDF model from atmospherically corrected reflectances. These albedo measures then are intrinsic properties of the surface. They represent the following two cases. The directionalhemispherical integral (4), called the "black-sky albedo," represents the case of single-beam irradiation in the absence of diffuse skylight, i.e., the situation of a perfectly clear sky. The bihemispherical albedo, called the "white-sky albedo," given by (6), with $\overline{\rho_{s}}$ replaced by $\rho_{b}$ and the integration over $\theta_{s}$, represents the case of perfectly diffuse illumination, i.e., the case of a perfectly turbid atmosphere, similar to an overcast situation. The albedo under conditions of atmospheric scattering of light will then be a value between these two extreme cases and will depend on the actual aerosol and water vapor loading.

It may be noted that the black-sky albedo is a function of solar zenith angle, whereas the white-sky albedo is a constant. It is an advantageous property of kernel-based models that the black-sky albedo may be formulated as a linear combination of the BRDF model parameters and the black-sky integrals of the kernels. Since these integrals may be precomputed and tabulated, the black-sky albedo can be generated from known model parameters at any solar zenith angle without numerical integration [Lewis, 1995] using a very compact lookup table that will be provided with the product.

Black-sky and white-sky albedo will be given in all seven MODIS land bands, spanning from 0.47 to $2.13 \mu \mathrm{m}$. They will also be derived in three broadbands, from 0.4 to $0.7 \mu \mathrm{m}$, 0.7 to $3.0 \mu \mathrm{m}$, and 0.4 to $3.0 \mu \mathrm{m}$, which are useful to climate modeling, where the wavelength domain is commonly partitioned in this manner [Dickinson, 1983]. In the shortwave region, vegetation dominantly absorbs radiation, whereas in the longerwave region, it is mainly scattered. Broadband albedos are not simply spectral averages of the albedo. They are defined such that given a downwelling broadband flux, the corresponding broadband upwelling flux is derived.

Narrowband-to-broadband albedo conversion will be performed by weighting the narrowband albedos by their associated proportion of downwelling solar irradiance. This method has been used with nadir Landsat measurements [Brest and Goward, 1987] and was shown to be satisfactory by Ranson et al. [1991], although Starks et al. [1991] report a case where a significant bias occurred. Since the downwelling solar irradiance depends on the atmospheric state during observation, there is an undesirable dependence of the broadband albedos on that state. We are currently considering resolving this situation by using the irradiance found from applying molecular scattering alone (clean-sky case). However, the spectral albedos will be provided by the MODIS BRDF/albedo product as well, so individual 
Table 3. Spectral-to-Broadband Albedo Conversion: Accuracy of Results Based on Seven-Band Splines As Compared to Exact Results

\begin{tabular}{lllllrrr}
\hline & \multicolumn{3}{c}{ Exact Results } & & \multicolumn{3}{c}{ Band-Based Deviations } \\
\cline { 2 - 4 } \cline { 6 - 8 } Cover & Total & Vis & IR & & Total & Vis & IR \\
Type & $0.4-2.2 \mu \mathrm{m}$ & $0.4-0.7 \mu \mathrm{m}$ & $\mathbf{0 . 7 - 2 . 2 \mu \mathrm { m }}$ & & $\mathbf{0 . 4 - 2 . 2 \mu \mathrm { m }}$ & $\mathbf{0 . 4 - 0 . 7 \mu \mathrm { m }}$ & $\mathbf{0 . 7 - 2 . 2 \mu \mathrm { m }}$ \\
\hline Grass & 0.22 & 0.058 & 0.34 & & $-0.9 \%$ & $+0.5 \%$ & $-1.2 \%$ \\
Soil & 0.18 & 0.10 & 0.24 & & $-0.4 \%$ & $-3.2 \%$ & $+0.4 \%$ \\
Snow & 0.84 & 0.99 & 0.72 & & $-2.1 \%$ & $-0.2 \%$ & $-4.0 \%$ \\
\hline
\end{tabular}

researchers will always be able to carry out their own conversions.

Table 3 shows sample results illustrating the potential accuracy achievable in narrowband-to-broadband albedo conversion using the seven MODIS land bands for three different land cover types. The broadband albedos derived from splines to the spectral values are within 1 or $2 \%$ of the real values.

\subsection{Sensitivity of BRDF and Albedo Retrievals to Angular Sampling and Noise}

The behavior of the Ambrals BRDF model under inversion was studied in detail under conditions of angular sampling as expected from MODIS and MISR. Two types of studies were carried out. The first is a study of the influence of random noise on the accuracy of the inversion. The second is a study of how well a BRDF derived from a limited set of angular reflectances interpolates and extrapolates to angles not observed. Both of these extensive studies will be reported in full detail elsewhere. Here, only a brief overview over the main results can be given.

Both the noise sensitivity and the interpolation/extrapolation accuracy study were conducted using simulated 16-day MODIS and MISR cloud-free viewing and illumination geometries simulated by the Xsatview software by Barnsley and Morris [Barnsley et al., 1994]. Investigations were carried out as a function of latitude and day of the year. The quantities studied were nadir-view reflectance and black-sky albedo at the prevailing mean Sun angle of observations ("interpolation") and for a fixed nadir or $10^{\circ}$ Sun zenith angle ("extrapolation"). White-sky albedo and the model parameters themselves were also investigated.

The noise sensitivity study was carried out by taking advantage of the linear mathematical properties of kerneldriven models that allow an analytical analysis following methods developed by Gauss [Whittaker and Robinson, 1960]. The diagonal elements of the inversion matrix found in the minimization problem, which depend only on the kernel values at the angles sampled, may be used to compute so-called "weights of determination," or "noise inflation factors" [Wanner et al., 1996]. These factors indicate how random uncertainty in the reflectances sampled translates into uncertainty in the BRDF and in albedo. Noise inflation factors depend on the sampling geometry alone, that is, for MODIS and MISR sampling they vary with latitude and time of year, but for kernel-based models, they do not depend on wavelength or the type of BRDF viewed. The study conducted shows that both interpolated and extrapolated nadir-view reflectances and black-sky albedos as well as the white-sky albedo have median noise inflation factors of less than 0.5 and worst values of less than about 1 , demonstrating that the inversions are stable with respect to random noise.

In the presence of clouds these factors are expected to increase with the square root of the number of observations, provided that the angular coverage of the samples does not change significantly. A comparison of the Ambrals noise sensitivity with the corresponding sensitivity of the semiempirical three-parameter BRDF model by Rahman et al. [1993] shows that both models behave very simi-

Table 4. Expected BRDF/Albedo Product Accuracy: Median Values and Ranges for 16-Day MODIS and MISR Sampling at Different Latitudes and Times of Year

\begin{tabular}{|c|c|c|c|}
\hline \multicolumn{2}{|c|}{ Quantity Investigated } & \multirow{2}{*}{$\begin{array}{c}\begin{array}{c}\text { Noise Inflation } \\
\text { Factor }\end{array} \\
0.21(0.18-0.28) \\
0.17(0.15-0.20)\end{array}$} & \multirow{2}{*}{$\begin{array}{c}\begin{array}{c}\text { Interpolation/Extrapolation } \\
\text { Error, \% }\end{array} \\
3.3(0.7-8.1) \\
3.1(0.5-9.6)\end{array}$} \\
\hline $\begin{array}{l}\text { Interpolation } \\
(\text { Sun zenith }= \\
\text { mean of obs.) }\end{array}$ & $\begin{array}{l}\text { nadir reflectance } \\
\text { black-sky albedo }\end{array}$ & & \\
\hline $\begin{array}{l}\text { Extrapolation } \\
(\text { Sun zenith }= \\
\left.\text { nadir } / 10^{\circ}\right)\end{array}$ & $\begin{array}{l}\text { nadir reflectance } \\
\text { black-sky albedo }\end{array}$ & $\begin{array}{l}0.45(0.17-1.08) \\
0.23(0.18-0.49)\end{array}$ & $\begin{array}{l}5.9(0.8-28.7) \\
4.8(0.8-16.0)\end{array}$ \\
\hline $\begin{array}{l}\text { Combined } \\
\text { (all Sun zeniths) }\end{array}$ & white-sky albedo & $0.31(0.17-0.82)$ & $6.0(1.4-14.2)$ \\
\hline
\end{tabular}

MISR, multi-angle imaging spectroradiometer. 
larly. The study was also carried out using MODIS-only and MISR-only sampling. Results demonstrated that the quality of a MODIS-only product is greatly enhanced by adding MISR data. The noise inflation factor may be used as a quantitative indicator of sampling quality in operational BRDF inversions and will be carried by the MODIS BRDF/albedo product.

For the interpolation and extrapolation error study, a three-dimensional discrete ordinate numerical BRDF forward model by Myneni et al. [1992] was used to create surface reflectances at the angles sampled by MODIS and MISR over 16 days. The study was carried out for BRDFs of six different biome types (grassland, shrubs, broadleaf crops, savanna, broadleaf forest, conifers) and for red and near-infrared wavelengths. Upon inversion of the Ambrals BRDF model results were compared with those known from the forward model. At the mean Sun zenith angle of the observations the predicted nadir view reflectance and black-sky albedo were found to have a median deviation from the true value of about 3 to $4 \%$. At a Sun zenith angle of $10^{\circ}$, reflectance showed a median deviation of about $6 \%$, whereas black-sky albedo was accurate to about $5 \%$. This shows that while interpolation can be carried out with good precision, extrapolation depends more on favorable sampling conditions. White-sky albedo could be determined with a median accuracy of $6 \%$. Since these numbers are the results of model-to-model comparisons, they should be interpreted with caution; problems can be due to either model. In cases with unfavorable sampling, errors of up to 10 or $20 \%$ may occur.

Table 4 summarizes the noise sensitivity and the interpolation/extrapolation study results. The results in both cases are medians over all cases investigated. The ranges given are chosen to include two thirds of all relevant data.

\section{Algorithm}

\subsection{BRDF/Albedo in the MODIS Production Chain}

The MODIS BRDF/albedo product is generated as part of the surface reflectance processing chain for the MODIS instrument, which runs from calibration and geolocation through atmospheric correction, gridding, and resampling to BRDF and albedo retrieval. The latter relies on atmospherically corrected surface reflectance data from both MODIS and MISR and atmospheric coupling descriptors for updating atmospheric corrections where necessary after the initial BRDF retrievals. Product generation is supported and gaps are filled by having available current land cover and topographic information, the BRDF/albedo results from the previous production cycle, and a global database of accumulated BRDF/albedo knowledge that will be built over time.

MODIS level-2 reflectances that have been cloud cleared and atmospherically corrected are binned into the MODIS level-3 grid over a period of 16 days and combined with all MISR observations acquired during that time. The data are averaged to a spatial resolution of $1 \mathbf{k m}$, where each observation is weighted by the respective overlap between the grid cell and the observation footprint. Quality information associated with the data is translated to quality coefficients that are used to weight individual observations in the subsequent BRDF inversion. This allows special consideration of data where, for example, atmospheric correction was difficult or aerosol information was taken from standard tables because no aerosol retrievals were available.

The directional observations thus assembled are then analyzed by inverting the five model variants listed in Table 2 to find the Ambrals kernel combination that describes the observations best. BRDF model inversion is carried out by straightforward matrix inversion [Lewis, 1995]. The four bands common to MODIS and MISR are inverted first. The model chosen from this analysis is then applied to the three remaining bands, in which only MODIS data are available. The model parameters found are then output along with extensive quality control data and other information necessary for an assessment of the product. Integration of the BRDF is carried out to provide black-sky and white-sky albedos. Use of a digital terrain database will allow correction for BRDF effects created by topographic shading after the launch of AM-1 when a suitable global dataset is available. Initially, the BRDF derived will characterize the combined BRDF of vegetation/soil and topography.

The Ambrals model kernel combination that best describes the observations is selected from the five available model variants as follows: Generally, the kernels to be used will be chosen to provide the fit with the smallest root bandaveraged mean squared error (RMSE) when inverting the available multiangular surface reflectances. The two cases, where an exception is made are if the RMSE found is above a pre-determined threshold, or the angular sampling coverage is poor for reliable inversion, either because observations span only a small angle range or the number of observations is too small. In these cases information from the supporting ancillary databases is used to limit the inversion. If angular sampling is good but the RMSE found is high, the kernel combination suggested by the ancillary data (previous BRDF, BRDF accumulated database, land cover type, topography) is used instead of the best fitting model variant if the resulting RMSE is not much worse. The model parameters are still derived from a full inversion of the reflectances.

If angular sampling is bad or the number of reflectances available is too small, a full inversion cannot be trusted and gaps in the product would result. In this case, the BRDF kernel combination and model parameters suggested by the ancillary databases will be used, but the magnitude of the BRDF (the isotropic constant of the model) will be adjusted to the observed reflectances. Through this the shape but not the magnitude of the BRDF will be fixed. This procedure also guarantees that the reflectance information available in cases not permitting a full BRDF inversion will not be simply lost but used to the extent possible.

In each case, the source of the resulting BRDF information will be recorded so that users can filter the output according to their needs. The ancillary database accumulating BRDF knowledge over time, keyed by season, and the relationships between land cover type and BRDF used will be established post launch from the observed data and updated at intervals.

Since the Ambrals model allows different kernels to be selected according to the specific land cover type under observation, the mathematical expressions used on adjacent 
pixels may be slightly different. While this is expected to provide the best BRDF and albedo information on a per pixel basis, it may also cause difficulties in mapping between pixels and where a much simpler uniform approach is desired. As a consequence, the MODIS BRDF/albedo product will always also provide the full inversion results for the modified Walthall BRDF model [Walthall et al., 1985; Nilson and Kuusk, 1989]. This model is purely empirical and is expected to produce results of reduced accuracy, particularly under conditions of sparse angular sampling. However, the simplicity of the mathematical expression used and the fact that it will be the same for all pixels are attractive to applications where a reduced accuracy is acceptable or where spatial consistency in the BRDF model is deemed important.

The product generation rules are as follows: If less than eight observations are available in a 16-day period, or they are clustered in a small region of angle space, this will be deemed insufficient for a full inversion. Angular coverage will be monitored through the determinant of the inversion matrix, which reflects the power of a given sampling to discriminate the model parameters. If all the looks are at very high zenith angles, processing continues, but the appropriate quality flags are set to indicate the situation. Similarly, the quality of the product will be lower if no MISR data are available. If the supporting inputs of land cover, digital terrain, and ancillary accumulated BRDF database are not available, processing continues with lower quality, since these inputs are supportive only. No BRDF is derived over oceans or inland water, although pixels with subresolution water content will be processed. For snow we expect the Ross kernels to apply, but this still needs to be investigated. Processing of areas covered by sea ice is currently under consideration where the solar zenith angle is large enough.

Details about the algorithm, its theoretical basis, and its dependence on other products may be found in the NASA Algorithm Technical Basis Document [Strahler et al., 1996b], available on the internet under the EOS site hierarchy.

\subsection{Output Product and Quality Flags}

BRDF inversion results will be given in the form of identifiers determining the Ambrals model kernel combination used for each pixel and the list of model parameters found for each band for the Ambrals and the modified Walthall model. Black-sky albedo at any solar zenith angle and white-sky albedo for both models can be constructed with little computational effort directly from the model parameters using a small lookup table of precomputed kernel integrals that will be provided to users with the product. Broadband albedos will be given in a similarly parameterized form.

Quality and product information will include the following: an overall quality indicator; a flag detailing the source of the BRDF given (new full inversion, limited inversion or taken from ancillary database); the quality of the fit over wavelengths; view and Sun angle coverage; the mean Sun angle of the observations; flags indicating whether topographic effects are expected in the BRDF, whether MISR data were available and whether atmospheric correction was updated; and the land/water and data availability mask. The RMSE of the inversions, a quantitative descriptor of the quality of the angular sampling found, and a parameter describing the relative contribution of surface and volume scattering to the scene are also recorded.

In addition to this 1-km BRDF/albedo product, a spatially degraded product at a quarter-degree resolution will be produced for direct use in climate models. This product will also carry information on the subgrid variability of albedo.

\subsection{Ambrals BRDF Inversion and Modeling Tool}

The operational version of the processing algorithm has been programmed to provide maximum efficiency by avoiding multiple evaluation of expressions shared by several kernels and through lookup table approaches to kernel value retrieval and albedo calculations. One 16-day BRDF/albedo product at full resolution and in all seven bands will have a total file size of approximately 35 GByte. This size also applies to the BRDF knowledge database to be created. The processing power required to produce the 16-day product in real time is currently estimated at about 300 million floating point operations per second. Preparing the input data for BRDF/albedo processing requires additional resources.

A user working with the product will need to be able to reconstruct the BRDF from the parametrization provided by the MODIS BRDF/albedo product. For this an algorithm is required that allows forward modeling of each of the models used in the inversion. The Ambrals code will be provided in a form that allows forward and inverse BRDF modeling of kernel-driven models, provides a selection of science options, numerical BRDF integration, and permits easy adding of new models. This code can also be used to degrade the product to coarser spatial resolutions and derive the corresponding albedos. The Ambrals code, together with a user guide, is presently available from the authors upon request for BRDF modeling work and preparation of BRDF-dependent algorithms.

\section{Validation}

\subsection{Validation Outline}

Validation of the BRDF/albedo product will be performed in three ways. First, the semiempirical BRDF models will be validated for as many types of land cover as possible to assure that they provide adequate mathematical descriptions of common BRDF shapes. This is being done using fieldmeasured, laboratory-measured, and numerically simulated BRDFs.

Second, large-scale BRDF and albedo retrievals need to be demonstrated given the constraints of sampling, atmospheric correction, and temporal composition of data occurring in a remote sensing situation. This is done primarily using multiangular data from airborne sensors and from AVHRR.

Third, the quality and accuracy of the product need to be monitored during postlaunch product generation. This validation will be part of a MODIS-wide and EOS-wide validation and quality assurance effort based on tower measurements, aircraft campaigns, and intercomparisons with the results obtained from other space-based sensors. 


\subsection{Model Validation}

Basic model validation was carried out by applying the Ambrals BRDF model to multiangle reflectance data measured in the field for a variety of land cover types. Here we demonstrate some examples of this work, selected to represent four distinct categories of land cover types: forests, barren or sparsely vegetated lands, grasses and grasslike crops, and broadleaf crops. The data sets used include two soybean data sets observed by Ranson et al. [1985] using an Exotech model 100 radiometer. They represent canopy coverages of 72 and $99 \%$ and solar zenith angle ranges from $20^{\circ}$ to $49^{\circ}$ and $31^{\circ}$ to $61^{\circ}$, respectively. Inversion was carried out using all four bands observed, including the red and nearinfrared bands. An aspen and a spruce forest data set were selected from measurements by Deering et al. [1995] using a PARABOLA instrument, and the red and near-infrared channel were used for inversions. The solar zenith angle range was $45^{\circ}$ to $59^{\circ}$ and $36^{\circ}$ to $59^{\circ}$, respectively. All other data were acquired by Kimes [1983] and Kimes et al. [1985, 1986] at solar zenith angles ranging from $25^{\circ}$ to $79^{\circ}$, varying from one data set to another. The whole of the viewing hemisphere was sampled for all data sets.

Evaluations were carried out for the red and near-infrared (NIR) channels. Table 5 lists the data sets, gives the model with the lowest root of the band-averaged squared deviation between modeled and observed reflectances (RMSE), and the coefficients of linear correlation between the modeled and observed reflectances in the red and in the NIR band. The RMSEs are found to be between 1.1 and $4.6 \%$, which is satisfactory, and the correlation coefficients commonly are larger than 0.8 or even 0.9 . These results are typical for those found for a number other data sets (including AVHRR, airborne POLDER, ASAS, and field-measured data) using variants of the Ambrals model used here [Privette and Ver- mote, 1995; Hu et al., 1996; White et al., 1996]. The corn data set has rather large irregular variations in the observed reflectance, which explains the low red band correlation coefficient for this one data set.

Figure 3 shows the correlation between observed and modeled reflectances for one example from each of the four land cover groups. Even though there are some deviations, the modeled reflectances generally follow the observed values. It should be noted that the unvegetated plowed field data set is described well by the geometric-optical theory of shadowing provided by the Li-sparse kernel. This is not unexpected. Geometric-optical theories are based on relative geometric proportions and thus are independent of the height-to-width ratio of the scattering objects. The plowed field consists of shadow-casting clumps of earth sitting on the ground, geometrically similar to the crowns of a relatively larger bush canopy. Note that in the cases of irrigated wheat and the plowed field there are some reflectances that are noticeably larger than the others and that these are modeled correctly.

Figure 4 shows the RMSE for each of five BRDF models tested for three examples of each land cover group (the Cox-Munk/Li-sparse model was not used since it is only applicable to scenes involving a water component). Obviously, all data sets are fitted well by at least one of the models. In a number of cases, one type of model fits the data better than another. Examples include the models containing the $\mathrm{Li}$ sparse kernel for the barren or sparsely vegetated land cover types, the Li-dense kernel for the hardwood forest, and the Ross-thick kernel for the orchard grass and the second soybean data set. In some other cases, all models provide good fits, as for the aspen data set, the grass lawn, and the corn. This may be explained by a low variance of reflectances in the particular angular ranges sampled, which places a large emphasis on the isotropic constant of the models. The empirical modified Walthall model performs fairly well in some

Table 5. Sample Ambrals Model Fit Accuracies Using Field-Measured BRDFs of Several Different Land Cover Types in the Red and NIR Bands

\begin{tabular}{|c|c|c|c|c|c|}
\hline Cover Type & Source & Best Fit Kernels & RMSE, \% & $r(\mathrm{red}, \%)$ & $r(\mathrm{NIR}, \%)$ \\
\hline \multicolumn{6}{|c|}{ Needleleaf and Broadleaf Forests } \\
\hline Dense hardwood forest & Kimes & Ross-thick/Li-dense & 3.0 & 0.90 & 0.89 \\
\hline Dense pine forest & Kimes & Ross-thin/Li-dense & 4.1 & 0.78 & 0.72 \\
\hline Aspen forest & Deering & Ross-thick/Li-sparse & 2.5 & 0.92 & 0.88 \\
\hline Spruce forest & Deering & Ross-thick/Li-sparse & 1.1 & 0.95 & 0.94 \\
\hline \multicolumn{6}{|c|}{ Barren, Soil, Sparse Vegetation } \\
\hline Plowed Field & Kimes & Ross-thick/Li-sparse & 1.6 & 0.98 & 0.98 \\
\hline Annual grass (coverage $<5 \%$ ) & Kimes & Ross-thick/Li-sparse & 2.3 & 0.95 & 0.88 \\
\hline Steppe grass (coverage 18\%) & Kimes & Ross-thick/Li-sparse & 2.4 & 0.89 & 0.92 \\
\hline \multicolumn{6}{|c|}{ Grasses, Grasslike Crops } \\
\hline Grass lawn & Kimes & Ross-thin/Li-dense & 4.6 & 0.72 & 0.84 \\
\hline Irrigated wheat & Kimes & Ross-thick & 3.7 & 0.91 & 0.92 \\
\hline Orchard grass & Kimes & Ross-thick/Li-sparse & 3.1 & 0.84 & 0.91 \\
\hline \multicolumn{6}{|c|}{ Broadleaf Crops } \\
\hline Soybeans (coverage $72 \%$ ) & Ranson & Ross-thick/Li-sparse & 1.7 & 0.75 & 0.89 \\
\hline Soybeans (coverage $99 \%$ ) & Ranson & Ross-thick & 1.1 & 0.91 & 0.93 \\
\hline Soybeans (coverage $90 \%$ ) & Kimes & Ross-thick/Li-sparse & 4.3 & 0.78 & 0.81 \\
\hline Com (coverage $25 \%$ ) & Kimes & Ross-thin/Li-dense & 2.8 & 0.47 & 0.75 \\
\hline
\end{tabular}

Sources: Deering, Deering et al. [1995]; Ranson, Ranson et al. [1985]; Kimes, Kimes [1983], Kimes et al. [1985, 1986]. 

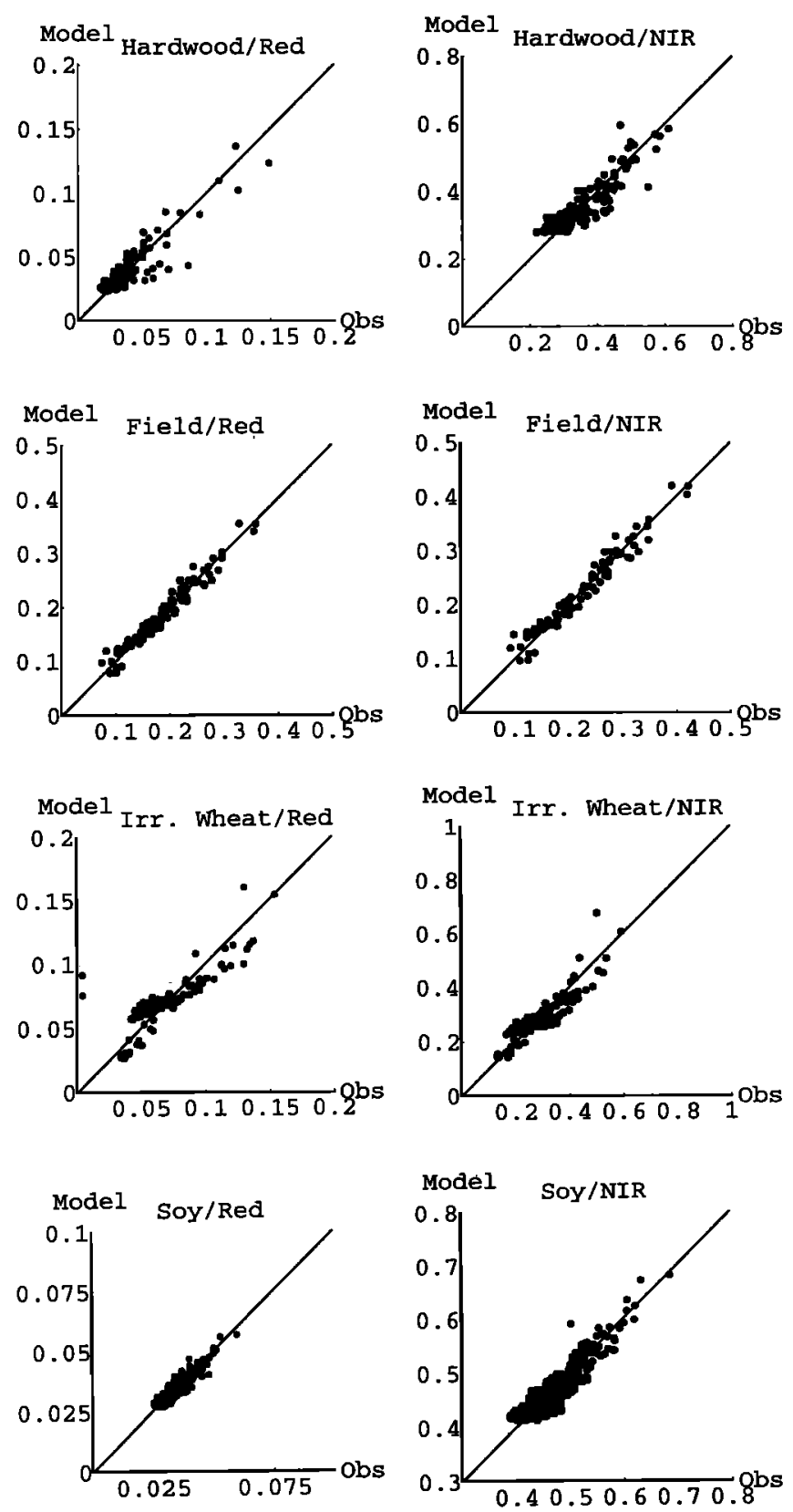

Figure 3. Observed versus modeled reflectances in the red and near-infrared bands for four distinct types of land cover. Forests are represented by a dense hardwood forest (coverage $79 \%$ ), barren terrain by a plowed field, grasslike vegetation by dense (irrigated) wheat (coverage $70 \%$, leaf area index 4.0), and broadleaf nonforest vegetation by a soybean data set (coverage $99 \%$, leaf area index 2.9). The first three data sets were observed by Kimes et al. [1985, 1986], the latter by Ranson et al. [1985]. In the first data set, the Li-dense kernel is predominant, in the second the Li-sparse kernel. In the third and fourth data sets the Ross-thick kernel is predominant.

cases, showing a lower RMSE than the best semiempirical model. However, this result is valid for the relatively good angular sampling that characterizes these validation data sets. Remotely sensed data will be much sparser in angle space, which is when the physical basis of the semiempirical models will give them an important advantage.
An important question is whether the differences in the RMSE found between model variants translate to noticeable differences in derived reflectances and albedos. If this were not the case, all model variants could be considered equally capable of modeling the BRDF of different land cover types, and using only one of them would be sufficient. However, there is indeed a difference between kernel combinations. Figure 5 shows this for two examples, the hardwood forest data and one of the soybean data sets. In the forest case, the models containing the Li-dense kernel clearly have a smaller RMSE, but the white-sky albedo is nearly the same for all model variants. This may be caused by the term $\cos \theta_{v} \sin \theta_{v} \cos \theta_{i} \sin \theta_{i}$ that occurs in the bihemispherical albedo integral. Values of the BRDF at very small and very large zenith angles, where the dependence of the BRDF on angle is strongest, have only a weak effect on the albedo. In the case of the soy data set, however, the opposite effect occurs. There is a noticeable difference in albedo between models based on the Ross-thin and those based on the Rossthick kernel even though the RMSEs of all model variants are similar.

The isotropic model constant $f_{i}$ (nadir Sun and view reflectance) shows, in this example, large differences between model variants for the case where the albedo varies little but only small variation in the case where the albedo differs across kernel combinations. These and similar findings for other data sets lead to the conclusion that choosing the correct model is important for generating accurate results. Using just one BRDF model or model variant will lead to results of varying quality for different land cover types. This situation will be aggravated under conditions of more limited sampling than is the case for these well-sampled field-measured data sets.

The feasibility of using kernel-based semiempirical models for modeling bidirectional reflectance data has also been shown by several authors using a model similar to the Ambrals model, employing the Ross-thick kernel and a geometric-optical kernel by Roujean et al. [1992]. Leroy and Roujean [1994] were able to correct noise-like temporal variations in AVHRR data that were due to varying angles of observations. Wu et al. [1995] successfully modeled AVHRR data for several different land cover types. Li et al. [1996] are able to remove mosaicking borders from an AVHRR normalized difference vegetation index (NDVI) image of a large area in Canada that were due to directional differences between overpasses. Privette and Vermote [1995] conclude that kernel-based models of the Ambrals model type perform satisfactorily for atmospheric correction purposes on an AVHRR desert scene. Barnsley et al. [1997a] apply the Roujean model and the modified Walthall model to multiangle images obtained by ASAS during the hydrology-atmosphere pilot experiment in the Sahel (HAPEX-Sahel). They show that the first parameter of the semiempirical model, which is the reflectance for nadir view and Sun, is the most stable parameter in the retrieval and matches spatially with the nadir-view images acquired. Maps of albedo are produced from the model inversions. Inversions of a soybean and a nasturtium data set observed in a Chinese laboratory that acquires rapid multiangle reflectance measurements has produced satisfactory results both when angular sampling was dense and when it was sparse [Strahler et al., 1995b]. 
Root Band-Averaged Mean Absolute Deviation of Model (RMSE)
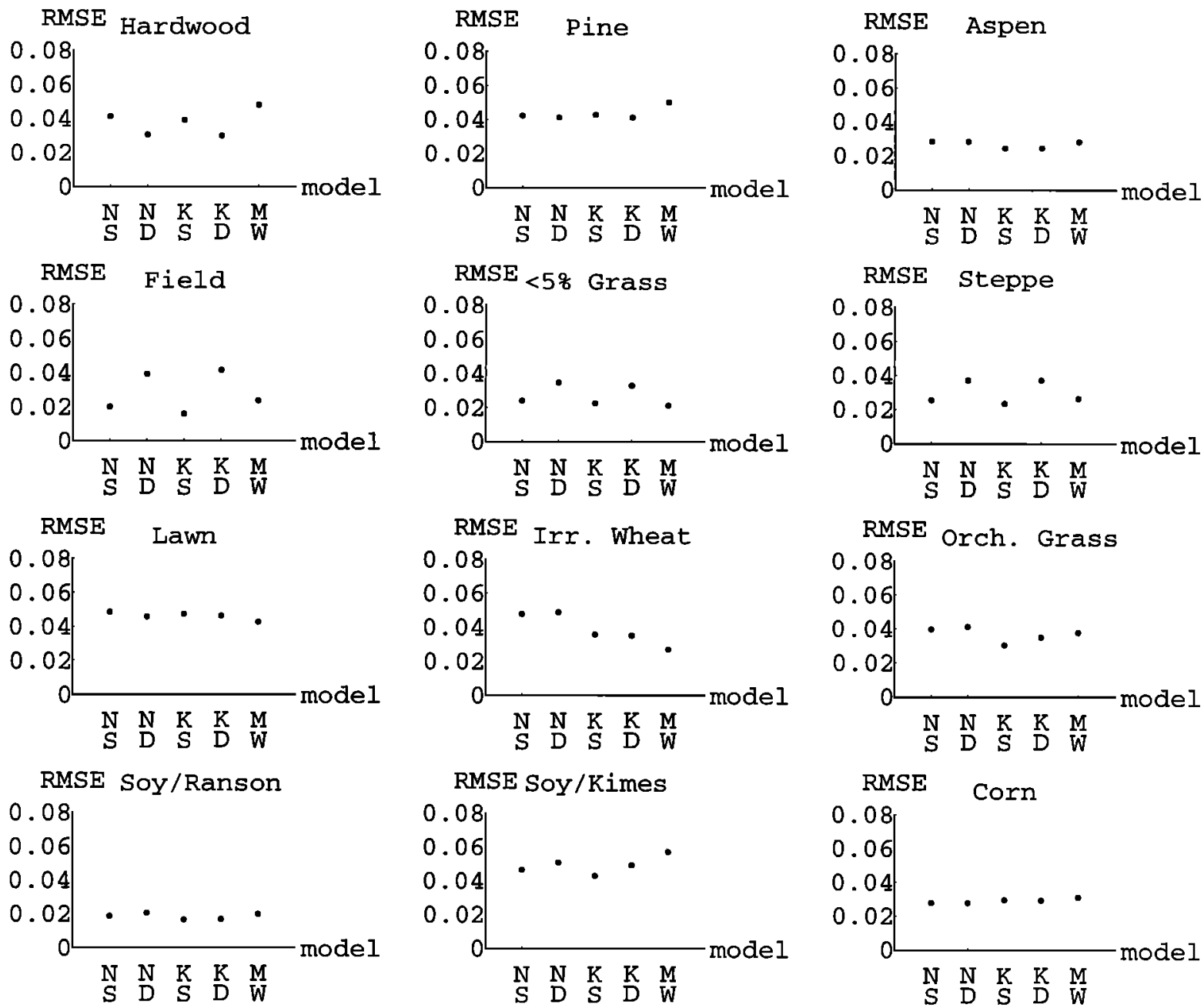

Figure 4. Root band-averaged mean square errors (RMSE) of fitting five kernel-based bidirectional reflectance distribution function (BRDF) model variants to bidirectional reflectance data measured by Kimes [1983], Kimes et al. [1985, 1986], Deering et al. [1995], and Ranson et al. [1985] for four different land cover types: top, forests; second row, barren or sparsely vegetated; third row, grasses and grasslike crops; bottom, broadleaf crops. Model codes: N, Ross-thin; K, Ross-thick; S, Li-sparse; D, Li-dense; MW, modified Walthall.

The conclusions to be drawn from these validation investigations are as follows: First of all, the kernel-driven semiempirical Ambrals BRDF model to be used in the MODIS BRDF/albedo algorithm is capable of fitting bidirectional reflectance data sets well. Second, different combinations of kernels are appropriate to different data sets, i.e., different land cover types. Using a small number of distinct kernel variants is therefore advisable if both albedo and BRDF are to be derived with low errors. Finally, the modified Walthall model, to be used as a uniform model parallel to the Ambrals model, is capable of fitting the data sets tested here as well, but it is expected to perform less well for inversion of the more limited angular sampling encountered from MODIS/MISR.

Additional field-measured BRDF data sets are expected to be available for continued model validation. The BRDF laboratory in Changchun, China, [Strahler et al., 1995b; Liang et al., 1997] will provide additional BRDF measurements in the future. Data acquired during the boreal ecosystems atmosphere study (BOREAS) campaigns are beginning to be available, such as the PARABOLA data sets by Deering et al. [1995], two of which were used in this paper. Artificial data sets have been created by Soffer et al. [1995] using model trees in a laboratory and by Lewis and Muller [1992] using Monte Carlo ray tracing. BRDF data sets generated numerically for several different biome types from a threedimensional radiative transfer code by Myneni et al. [1992] are being employed for a detailed study of model properties that will be reported elsewhere.

\subsection{Relationship of BRDF to Land Cover}

In Figure 4 there is an indication of distinctive patterns characterizing each land cover type group. In the case of the hardwood forest, clearly the two models involving the 
RMSE, White-Sky Albedo (Red and NIR), and

Isotropic Reflectance Constant (Red and NIR)
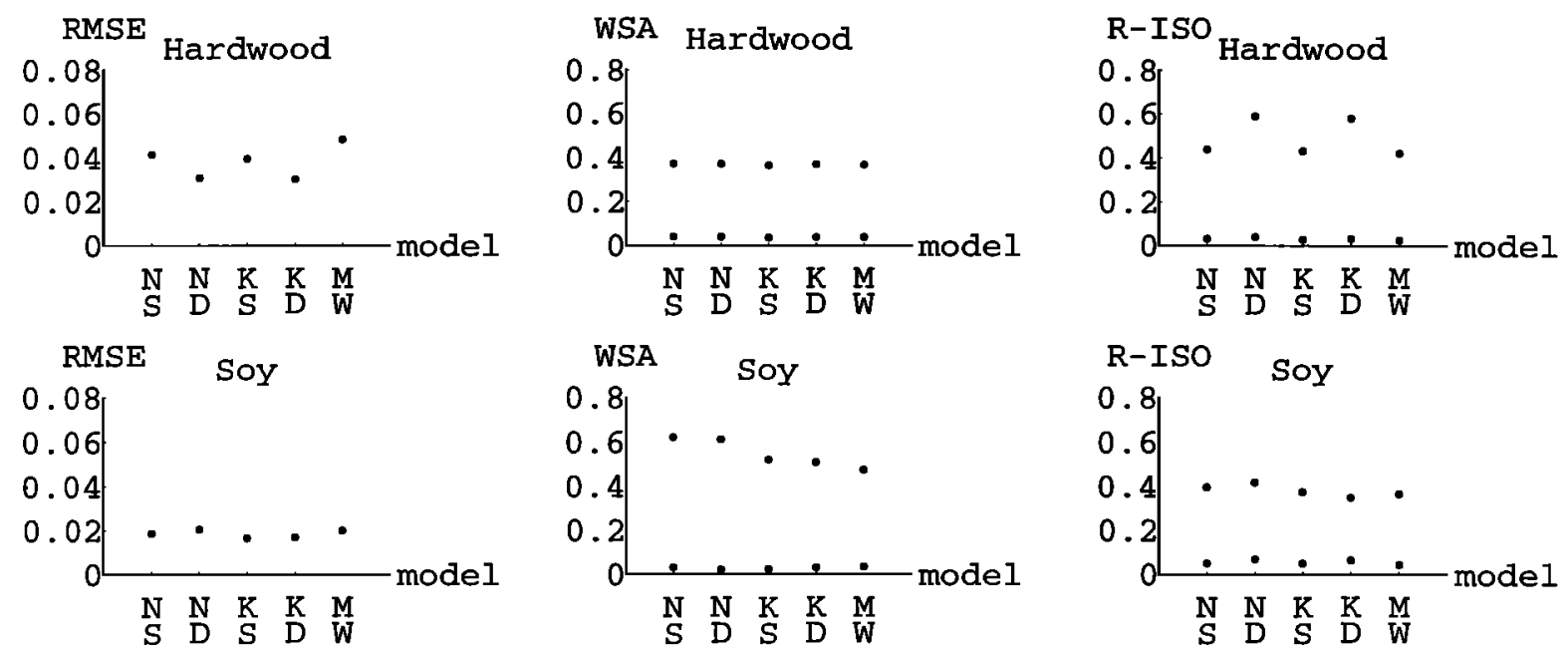

Figure 5. Root band-averaged mean square errors (RMSE), white-sky albedo (WSA) and the isotropic model constant $f_{i}$ (nadir Sun and view reflectances, R-ISO) of five kernel-based BRDF model variants to bidirectional reflectance data measured by Kimes et al. [1986] and Ranson et al. [1985] for two different land cover types: forest and broadleaf crop. Model codes: N, Ross-thin; K, Ross-thick; S, Li-sparse; D, Li-dense; MW, modified Walthall.

Li-dense kernel are much favored over those involving the Li-sparse kernel. This corresponds to the fact that the forest observed was indeed dense in nature. There is little difference between the model using the Ross-thick kernel and the model using the Ross-thin kernel. This may be explained by the fact that scattering is dominated by geometric optics, leading to a small weight given to the volume-scattering kernel. It does not matter which one is chosen. The case is less clear for the pine and the aspen data sets, but the aspen does show a slight improvement of fits if the Ross-thick kernei is used. Although it might well be expected from the scene type, this data set lacks a distinct hotspot, perhaps due to instrument characteristics.

All the examples given for barren or sparsely vegetated covers show a distinct preference for the Li-sparse kernel, which is driven by shadowing. The plowed field consisted of clumps of earth on the ground, leading to strong surfacescattering effects. The annual grass data set was acquired at a site with less than $5 \%$ of vegetation cover, where $40 \%$ of the surface was covered by stones and pebbles [Kimes and Sellers, 1985]. The steppe grass site had clumps of grass, and the total cover was $18 \%$. Obviously, the structured surface of these land covers is best represented by strong shadow-driven geometric-optical scattering.

Two of the three data sets given for dense grasses and grasslike crops show a preference for models containing the Ross-thick kernel, as might be expected for dense horizontally layered canopies that show little shadowing. In the broadleaf crops group, two of the three examples given are represented well by all models. They lack angular variation in the angle intervals sampled, resulting in a strong isotropic contribution to overall fitting that renders the contributions from the respective kernels less important. One set, how- ever, shows the influence of both shadowing and volume scattering in being modeled best by the Ross-thick/Li-dense model.

We think that there is an indication in these data that, where mutual shadowing of crowns plays a role, as in most forests, models containing the Li-dense kernel will be chosen, whereas in scenes where shadows are dominant, models using the Li-sparse kernel are preferred. These scenes would then be sparse forests or brushlands and rock-strewn deserts, rough (plowed) terrain, or clumped vegetation, respectively. When the Ross-thick kernel is chosen over the Ross-thin kernel, or all models perform well, a densely-layered canopy with no individual crowns is observed. The Ross-thin kernel is expected to be applicable to scenes with a thin but horizontally continuous layer of leaves over a rather solid background, which can be the ground or dense underlying vegetation.

Table 6 summarizes these correspondences in a tentative list of land covers likely to be associated with models containing specific kernels. Whether the relationships speculated upon here hold with any consistency and whether the differences are distinct enough to allow reliable inference of surface structural properties remains to be seen when much more extensive data sets than are now available can be studied. However, the fact that surface structure determines the angular dependence of the reflectance, as is known from physical reflectance forward modeling, should make a retrieval of at least general structural information of the type discussed here possible [Goel, 1989; Pinty and Verstraete, 1991]. If the classes obtained from such an analysis are not congruent with the classes derived from spectral land cover classifications, the combination of directional and spectral information should allow for more accuracy in land cover 
Table 6. Expected Relationship Matrix of Ambrals Kernels to Land Cover Types

\begin{tabular}{|c|c|c|c|}
\hline $\begin{array}{l}\text { Models Composed of } \\
\text { the Following Two } \\
\text { Kemels (right+down): }\end{array}$ & $\begin{array}{l}\text { Low Weight for } \\
\text { Li-Kernels: No Discrete- } \\
\text { Crown/Object Scattering }\end{array}$ & $\begin{array}{l}\text { Li-Sparse Kernel: } \\
\text { Scattering From Sparse } \\
\text { Discrete Crowns/Objects }\end{array}$ & $\begin{array}{l}\text { Li-Dense Kernel: } \\
\text { Scattering From Dense } \\
\text { Discrete Crowns/Objects }\end{array}$ \\
\hline $\begin{array}{l}\text { Low weight for } \\
\text { Ross-kernels: no } \\
\text { leaf-layer type } \\
\text { scattering }\end{array}$ & Lambertian surface & $\begin{array}{l}\text { plowed fields, } \\
\text { rock-strewn lands, } \\
\text { rugged topography; } \\
\text { mosaics of these with } \\
\text { sparse woodlands }\end{array}$ & dense buildings \\
\hline $\begin{array}{l}\text { Ross-thin kernel: } \\
\text { weak scattering from } \\
\text { layered leafs }\end{array}$ & $\begin{array}{l}\text { sparse uniform } \\
\text { grass }\end{array}$ & $\begin{array}{l}\text { sparse woodlands or } \\
\text { brush, no understory, } \\
\text { deciduous forests in } \\
\text { winter, young } \\
\text { broadleaf crops }\end{array}$ & $\begin{array}{l}\text { forests, dense brush; } \\
\text { mosaics of these with } \\
\text { sparse grass }\end{array}$ \\
\hline $\begin{array}{l}\text { Ross-thick kernel: } \\
\text { strong scattering from } \\
\text { layered leafs }\end{array}$ & $\begin{array}{l}\text { grasses, } \\
\text { grasslike crops }\end{array}$ & $\begin{array}{l}\text { sparse woodlands or } \\
\text { brush with understory, } \\
\text { broadleaf crops, } \\
\text { clumps of vegetation; } \\
\text { mosaic of grasses or } \\
\text { other dense layered } \\
\text { vegetation and trees }\end{array}$ & $\begin{array}{l}\text { grasses, } \\
\text { dense vegetation; } \\
\text { dense forests; } \\
\text { mosaics of these }\end{array}$ \\
\hline $\begin{array}{l}\text { Cox-Munk kernel: } \\
\text { specular surface }\end{array}$ & water, ice & $\begin{array}{l}\text { subpixel water, ice, } \\
\text { irrigated fields with } \\
\text { sparse forest, brush } \\
\text { or rugged topography; } \\
\text { rough ice surfaces }\end{array}$ & N/A \\
\hline
\end{tabular}

classification. An example is the distinction between dense and sparse forests, or between dense brush and sparse forest, which may have similar spectral properties but distinct bidirectional characteristics. A study by Barnsley et al. [1997b] shows that while the spectral information content of a scene of arable farmland obtained with the airborne multispectral scanner Daedalus is greater than the directional information content, the multiangle properties of the data nevertheless provide an important means of distinguishing between the occurring land cover types. For this reason, the MODIS land cover product will make use of BRDF-derived information where feasible [Strahler at al., 1996a].

\subsection{Prelaunch Product Prototyping}

Several projects are close to completion that apply the Ambrals BRDF model to AVHRR data over large regions, demonstrating and testing the MODIS algorithm. It is beyond the scope of this paper to report this work in detail. A study by $d$ 'Entremont et al. [1996] is targeted at inversions of AVHRR multiangle imagery for the whole of New England. Maps of albedo and kernel combinations chosen have been derived. A project conducted by Lewis includes largearea AVHRR data inversions for different times of the year for the Sahel region of Niger, Africa. A similar investigation, led by $\mathrm{X} . \mathrm{Li}$, is under way for a large area surrounding the site of Three Gorges dam project on the Yangtse River in China. These scientific studies, which also serve to prototype the MODIS BRDF/albedo product, demonstrate the feasibility of the approach and have been successful in generating BRDF and albedo images of large areas. Validation of the results obtained is the next step. In the Sahel region, for example, both ground-measured and airborne sensor multiangular data have also been evaluated for comparison.

Other sources of large-area data that will be used when they become available are data from the POLDER instrument, launched in August 1996, and the SPOT-VEGETATION sensor, also to be launched before the AM-1 platform. These data sets, observed from space, will be complemented by more limited data sets acquired from airborne sensors. Here, several campaigns have been carried out and will be carried out in the future with the ASAS, the MODIS airborne simulator (MAS), the compact airborne spectrographic imager (CASI), and the airborne POLDER sensors over a variety of sites. Work on processing these data is under way. Semiempirical models will be and are being applied to these data sets to optimize the algorithm for deriving BRDF and albedo from MODIS/MISR. AirMISR is a MISR-like instrument that will be very relevant to BRDF studies when it is operational in 1997.

\subsection{Postlaunch Product Validation}

In the postlaunch phase, a coordinated effort for validating the radiometric products of MODIS and EOS as a whole will be undertaken. Validation of BRDF and albedo is tied into validation of calibration, atmospheric correction, and several higher-order products. This effort is currently being developed in the EOS project.

A number of tower sites will be equipped with radiometers monitoring upwelling and downwelling radiances on a continuous basis. At a minimum these sensors will record broadband hemispherical fluxes. Spectral measurements will be made whereever possible. A selected number of sites will 
also feature directional measurements. The status of the land cover around the towers and atmospheric properties over the towers will be measured simultaneously. Tower sites will be chosen to represent major land cover types. One problem with many current sites observing aerosols, for example, is that little attention is given to the upward fluxes coming back from the surface (e.g., rooftop instruments will not do).

The observations obtained at a tower site will be scaled to the footprint size of the space-based sensor through occasional aircraft overflights that relate site properties to surrounding areas. Additionally, sites will be chosen to be homogeneous in land cover type. Scaling may also be inferred from using data from sensors with different resolutions and at different solar zenith angles such as geostationary satellites.

An important part of postlaunch product monitoring will also be cross-comparing the MODIS BRDF/albedo product with the corresponding MISR surface product and the POLDER BRDF and albedo product. Use of comparable data from meteorological satellites for validation purposes will also be investigated.

\section{Conclusions}

Surface reflectance is a key quantity in optical remote sensing. A multitude of derived parameters and estimates are based on surface reflectance, for example, land cover classifications, snow and ice maps, and vegetation state parameters. However, since the reflectance of the land surface is anisotropic in nature, retrieval of the bidirectional reflectance distribution function is essential for an exact interpretation of the data and for characterizing average reflectance in the form of albedo measures. In addition, BRDF and albedo are required for accurate computations of radiative transport in the atmosphere, providing the directional characteristics and the magnitude of scattering at the lower boundary. This is of importance in atmospheric correction and in the radiation transfer schemes of climate and weather models.

The MODIS BRDF/albedo product will provide this information to a wide variety of users as part of the EOS-MODIS standard product catalog. The expected uses comprise correction of images for directional effects; standardizing images to common viewing and illumination geometries; deriving vegetation indexes free of directional effects; providing lower boundaries for atmospheric correction and radiation budget investigations; retrieving precise measures of land surface albedo; inferring land surface structural characteristics, especially of vegetation and topography; and as an input to land cover classification. A possible future application is in cloud detection.

The MODIS BRDF/albedo product will employ the Ambrals kernel-based semiempirical BRDF model, making use of a total of five different available kernel functions, and the modified Walthall model for modeling the multiangle data. These models have been shown to fit a variety of observed multiangular data sets well. Besides possessing beneficial computational qualities, such as analytical inversion, direct spatial scaling and generic accommodation of mixed pixels, their physical basis allows for credible extrapolation of the BRDF to angles not covered by the observations. The
BRDFs found will be integrated to provide integral measures of albedo that do not depend on atmospheric state.

The combination of these activities, it is hoped, will contribute to the goal of EOS, to further the understanding of the Earth system in a time of potentially large changes induced by human activity. The production of a routine global BRDF and albedo product will lead to an increased understanding of the role that directional anisotropy in the reflectance of the Earth's surface plays in the global energy system.

Acknowledgments. We would like to thank Jordan Borak for generating the data for the narrowband-to-broadband conversions shown in Table 3, Baojin Zhang for work on model inversion accuracy under varying angular sampling, and Jim Tallent for work on the Ambrals code. This work was supported by NASA under NAS5-31369, and in the UK by NERC under the TIGER-SVATS program and the European Union under the 3rd Framework Human Capital and Mobility program.

\section{References}

Barnsley, M. J., A. H. Strahler, K. P. Morris, and J.-P. Muller, Sampling the surface bidirectional reflectance distribution function (BRDF): Evaluation of current and future satellite sensors, Remote Sens. Rev., 8, 271-311, 1994.

Barnsley, M. J., D. Allison, and P. Lewis, On the information content of multiple-view-angle (MVA) images, Int. J. Remote Sens., in press, 1997a.

Bamsley, M. J., P. Lewis, M. Sutherland, and J.-P. Muller, Estimating land surface albedo in the HAPEX-Sahel southern super-site: Inversion of two BRDF models against multiple angle ASAS images, J. Hydrol., in press, 1997b.

Brest, C. L., and S. N. Goward, Deriving surface albedo measurements from narrow band satellite data, Int. J. Remote Sens., 8, 351-367, 1987.

Cihlar, J., D. Manak, and N. Voisin, AVHRR bidirectional reflectance effects and compositing, Remote Sens. Environ., 48, 77-88, 1994.

Cox, C., and W. H. Munk, The measurement of the roughness of the sea surface from photographs of the Sun glitter, J. Opt. Soc. Am., 44, 838-850, 1954.

Deering, D. W., S. P. Ahmad, T. F. Eck, and B. P. Banerjee, Temporal attributes of the bidirectional reflectance for three boreal forest canopies, Proc. Int. Geosci. Remote Sens. Symp., 95, 1239-1241, 1995.

d'Entremont, R. P., C. B. Schaaf, and A. H. Strahler, Cloud detection and land surface albedos using visible and near-infrared bidirectional reflectance distribution models, Proc. 8th Conf. Satell. Meteorol. Oceanogr., 334-338, 1996.

Deschamps, P. Y., F. M. Breon, M. Leroy, A. Podaire, A. Bricaud, J. C. Buriez, and G. Seze, The POLDER mission: Instrument characteristics and scientific objectives, IEEE Trans. Geosci. Remote Sens., 32, 598-615, 1994.

Dickinson, R. E., Land surface processes and climate-surface albedos and energy, Adv. Geophys., 25, 305-353, 1983.

DiGirolamo, L., and R. Davies, A band-difference angular signature technique for cimus cloud detection, IEEE Trans. Geosci. Remote Sens., 32, 890-896, 1994.

Diner, D., C. J. Bruegge, J. V. Martonchik, G. W. Bothwell, E. D. Danielson, V. G. Ford, L. E. Hovland, K. L. Jones, and M. L. White, A multi-angle image spectroradiometer for terrestrial remote sensing with the Earth Observing System, Int. J. Imag. Syst. Technol., 3, 92-107, 1991.

Goel, N. S., Inversion of canopy reflectance models for estimation of biophysical parameters from reflectance data, in Theory 
and Applications of Optical Remote Sensing, pp. 205-251, John Wiley, New York, 1989.

Gutman, G., Normalization of multi-annual global AVHRR reflectance data over land surfaces to common Sun-target-sensor geometry, Adv. Space Res., 14(1), 121-124, 1994.

Henderson-Sellers, A., and M. F. Wilson, Surface albedo for climate modeling, Rev. Geophys., 21, 1743-1778, 1983.

$\mathrm{Hu}, \mathrm{B}$. , W. Wanner, X. Li, and A. Strahler, Validation of kerneldriven semiempirical BRDF models for application to MODISMISR data, Proc. Int. Geosci. Remote Sens. Symp., 96, 1669$1671,1996$.

Kaufman, Y. J., The atmospheric effect on remote sensing and its correction, in Theory and Applications of Optical Remote Sensing, pp. 336-428, John Wiley, New York, 1989.

Kimes, D. S., Dynamics of directional reflectance factor distribution for vegetation canopies, Appl. Opt., 22(9), 1364-1372, 1983.

Kimes, D. S., and P. J. Sellers, Inferring hemispherical reflectance of the Earth's surface for global energy budgets from remotely sensed nadir or directional radiance values, Remote Sens. Environ., 18, 205-223, 1985.

Kimes, D. S., W. W. Newcomb, C. J. Tucker, I. S. Zonneveldt W. van Wijngaarden, J. de Leeuw, and G. F. Epema, Directional reflectance factor distributions for cover types of Northern Africa, Remote Sens. Environ., 18, 1-19, 1985.

Kimes, D. S., W. W. Newcomb, R. F. Nelson, and J. B. Schutt, Directional reflectance distributions of a hardwood and a pine forest canopy, IEEE Trans. Geosci. Remote Sens., 24, 281-293, 1986.

Kriebel, K. T., Measured spectral bidirectional reflection properties of four vegetated surfaces, Appl. Opt., 17(2), 253-259, 1978.

Kustas, W. P., R. D. Jackson, and G. Asrar, Estimating surface energy-balance components from remotely sensed data, in Theory and Applications of Optical Remote Sensing, pp. 604-627, John Wiley, New York, 1989.

Lee, T. Y., and Y. J. Kaufman, Non-Lambertian effects in remote sensing of surface reflectance and vegetation index, IEEE Trans. Geosci. Remote Sens., 24, 699-708, 1986.

Leroy, M., and J.-L. Roujean, Sun and view angle corrections on reflectances derived from NOAA/AVHRR data, IEEE Trans. Geosci. Remote Sens., 32, 684-697, 1994.

Leroy, M., J. L. Deuze, F. M. Breon, O. Hautecoeur, M. Herman, J. C. Buriez, D. Tanre, S. Bouffies, P. Chazette, and J.-L. Roujean, Retrieval of atmospheric properties and surface bidirectional reflectances over land from POLDER/ADEOS, J. Geophys. Res., this issue.

Lewis, P., The utility of kemel-driven BRDF models in global BRDF and albedo studies, Proc. Int. Geosci. Remote Sens. Symp., 95, 1186-1187, 1995.

Lewis, P., and M. J. Barnsley, Influence of sky radiance distribution on various formulations of Earth surface albedo, Proc. 6th Int. Symp. Phys. Meas. Sign. Remote Sens., 707-715, 1994.

Lewis, P., and J.-P. Muller, The advanced radiometric ray-tracer: ARARAT for plant canopy reflectance simulation, Proc. 29 th Conf. Int. Soc. Photogramm. Remote Sens., 26-34, 1992.

Li, X., and A. H. Strahler, Geometric-optical bidirectional reflectance modeling of the discrete crown vegetation canopy: Effect of crown shape and mutual shadowing, IEEE Trans. Geosci. Remote Sens., 30, 276-292, 1992.

Li, Z., and L. Garand, Estimation of surface albedo from space: A parametrization for global application, J. Geophys. Res., 99, 8335-8350, 1994.

Li, Z., J. Cihlar, X. Zheng, L. Moreau, and H. Ly, The bidirectional effects of AVHRR measurements over boreal regions, IEEE Trans. Geosci. Remote Sens., 34, 1308-1322, 1996.

Liang, S., A. H. Strahler, Q. Zhu, and X. Jin, Comparisons of radiative transfer models of vegetation canopies and laboratory measurements, IEEE Trans. Geosci. Remote Sens., in press, 1997.

Moody, A., and A. H. Strahler, Characteristics of composited
AVHRR data and problems in their classification, Int. J. Remote Sens., 15, 3473-3491, 1994.

Myneni, R. B., G. Asrar, and F. G. Hall, A three-dimensional radiative transfer method for optical remote sensing of vegetated land surfaces, Remote Sens. Environ., 41, 105-121, 1992.

Nicodemus, F. E., J. C. Richmond, J. J. Hsia, I. W. Ginsberg, and T. Limperis, Geometrical considerations and nomenclature for reflectance, Natl. Bur. Stand. Rep., NBS MN-160, 52 pp., 1977.

Nilson, T., and A. Kuusk, A reflectance model for the homogeneous plant canopy and its inversion, Remote Sens. Environ., 27, 157167, 1989.

Pinty, B., and M. M. Verstraete, Extracting information on surface properties from bidirectional reflectance measurements, $J$. Geophys. Res, 96, 2865-2874, 1991.

Pinty, B., M. M. Verstraete, and R. E. Dickinson, A physical model for predicting bidirectional reflectance over bare soil, Remote Sens. Environ., 27, 273-288, 1989.

Privette, J. L., and E. F. Vermote, Fitting remote sensing data with linear bidirectional reflectance models, Proc. SPIE Symp. Satell. Remote Sens., 2586, 172-179, 1995.

Rahman, H., B. Pinty, and M. M. Verstraete, Coupled surfaceatmosphere reflectance (CSAR) model, 2, Semiempirical surface model usable with NOAA advanced very high resolution radiometer data, J. Geophys. Res., 98, 20,791-20,801, 1993.

Ranson, K. J., L. L. Biehl, and M. E. Bauter, Variation in spectral response of soybeans with respect to illumination, view and canopy geometry, Int. J. Remote Sens., 6, 1827-1842, 1985.

Ranson, K. J., J. R. Irons, and C. S. T. Daughtry, Surface albedo from bidirectional reflectance, Remote Sens. Environ., 35, 201$211,1991$.

Ross, J. K., The Radiation Regime and Architecture of Plant Stands, 392 pp., Dr. W. Junk, Norwell, Mass., 1981.

Roujean, J. L., M. Leroy, and P. Y. Deschamps, A bidirectional reflectance model of the Earth's surface for the correction of remote sensing data, J. Geophys. Res., 97, 20,455-20,468, 1992.

Running, S. W., et al., Terrestrial remote sensing science and algorithms planned for EOS/MODIS, Int. J. Remote Sens., 15, 3587-3620, 1994

Running, S. W., E. R. Hunt, R. Nemani, and J. Glassy, MODIS leaf area index and fraction photosynthetically active radiation: Algorithm theoretical basis document, NASA EOS-MODIS Doc., 21 pp., 1995.

Sellers, P. J., Remote sensing of the land surface for studies of global change, NASA/GSFC Int. Satell. Land Surface Climatol. Proj. Rep., Columbia, MD, 1993.

Sellers, P. J., C. J. Tucker, G. J. Collatz, S. O. Los, C. O. Justice, D. A. Dazlich, and D. A. Randall, A global 1 degree by 1 degree NDVI data set for climate studies, 2 , The generation of global fields of terrestrial biophysical parameters from NDVI, Int. $J$. Remote Sens., 15, 3519-3545, 1994.

Soffer, R. J., J. R. Miller, W. Wanner, and A. Strahler, Winter boreal forest canopy BRF results: Comparisons between airborne data, laboratory simulations and geometrical-optical model data, Proc. Int. Geosci. Remote Sens. Symp., 95, 800-802, 1995.

Starks, P. J., J. M. Norman, B. L. Blad, E. A. Walter-Shea, and C. L. Walthall, Estimation of shortwave hemispherical reflectance (albedo) from bidirectionally reflected radiance data, Remote Sens. Environ., 38, 123-134, 1991.

Strahler, A. H., Vegetation canopy reflectance modeling - Recent developments and remote sensing perspectives, Proc. 6th Int. Symp. Phys. Meas. Sign., 593-600, 1994.

Strahler, A. H. X. Li, S. Liang, J.-P. Muller, M. J. Bamsley, and P. Lewis, MODIS BRDF/albedo product: Algorithm technical basis document, NASA EOS-MODIS Doc., version 2.1, 55 pp., 1994.

Strahler, A. H., M. J. Barnsley, R. d'Entremont, B. Hu, P. Lewis, X. Li, J.-P. Muller, C. B. Schaaf, W. Wanner, and B. Zhang, MODIS BRDF/albedo product: Algorithm theoretical basis document, NASA EOS-MODIS Doc. incl. Update, version 3.2, 65 pp., 1995a. 
Strahler, A. H., W. Wanner, Q. Zhu, and X. Jin, Bidirectional reflectance modeling of data from vegetation obtained in the Changchun Solar Simulation Laboratory, Proc. Int. Geosci. Remote Sens. Symp., 95, 1965-1967, 1995 b.

Strahler, A. H., J. T. Townshend, D. Muchoney, J. Borak, M. Friedl, S. Gopal, A. Hyman, A. Moody, and E. Lambin, MODIS land cover and land cover change: Algorithm technical basis document, NASA EOS MODIS Doc., version 4.1, 102 pp., 1996a.

Strahler, A. H., W. Wanner, C. B. Schaaf, X. Li, B. Hu, J.-P. Muller, P. Lewis, and M. J. Bamsley, MODIS BRDF/albedo product: Algorithm theoretical basis document, NASA EOS-MODIS Doc. incl. Update, version 4.0, 252 pp., $1996 \mathrm{~b}$.

Vermote, E. F., L. A. Remer, C. O. Justice, Y. J. Kaufman, and D. Tanre, MODIS atmospheric correction algorithm: Spectral reflectances, algorithm technical basis document, NASA EOS MODIS Doc., version 2.0, 42 pp., 1995.

Vermote, E. F., D. Tanre, J. L. Deuze, M. Herman, and J. J. Morcrette, Second simulation of the satellite signal in the solar spectrum: an overview, IEEE Trans. Geosci. Remote Sens., in press, 1997.

Vermote, E. F., N. El Saleous, C. O. Justice, Y. F. Kaufman, J. L. Privette, L. Remer, J. C. Roger, and D. Tanre, Atmospheric correction of visible to middle infrared EOS-MODIS data over land surfaces: Background, operational algorithm, and validation, $J$. Geophys. Res., this issue.

Walthall, C. L., J. M. Norman, J. M. Welles, G. Campbell, and B. L. Blad, Simple equation to approximate the bidirectional reflectance from vegetation canopies and bare soil surfaces, $A p p l$. Opt., 24, 383-387, 1985.

Wanner, W., X. Li, and A. H. Strahler, On the derivation of kemels for kemel-driven models of bidirectional reflectance, J. Geophys. Res., 100, 21077-21090, 1995a.

Wanner, W., A. H. Strahler, J.-P. Muller, M. Bamsley, P. Lewis, X. Li, and C. L. Barker Schaaf, Global mapping of bidirectional reflectance and albedo for the EOS MODIS project: The algorithm and the product, Proc. Int. Geosci. Remote Sens. Symp., 95, 525-529, 1995 b.

Wanner, W., P. Lewis, and J.-L. Roujean, The influence of directional sampling on bidirectional reflectance and albedo retrieval using kernel-driven models, Proc. Int. Geosci. Remote Sens. Symp., 96, 1408-1410, 1996.
White, H. P., R. Soffer, J. R. Miller, and W. Wanner, Semiempirical modeling of bidirectional reflectance utilizing the MODIS BRDF/albedo algorithm models, Proc. Int. Geosci. Remote Sens. Symp., 96, 1411-1413, 1996.

Whittaker, E., and G. Robinson, The Calculus of Observations, 397 pp., Blackie, Glasgow, 1960.

Wu, A., Z. Li, and J. Cihlar, Effects of land cover type and greenness on advanced very high resolution radiometer bidirectional reflectances: Analysis and removal, J. Geophys. Res., 100, 9179_ $9192,1995$.

Wylie, D. P., and W. P. Menzel, Two years of cloud cover statistics using VAS, J. Clim., 2, 380-392, 1989.

Wylie, D. P., W. P. Menzel, H. M. Woolf, and K. I. Strabala, Four years of global cirrus cloud statistics using HIRS, J. Clim., 7 , 1972-1986, 1994.

Zhang, Y.-C., W. B. Rossow, and A. A. Lacis, Calculation of surface and top of atmosphere radiative fluxes from physical quantities based on ISCCP data sets, 1, Method and sensitivity to input data set uncertainties, J. Geophys. Res., 100, 1149-1165, 1995.

C. L. Barker Schaaf, B. Hu, X. Li, A. H. Strahler, W. Wanner, Department of Geography and Center for Remote Sensing, Boston University, 725 Commonwealth Avenue, Boston, MA 02215. (email: schaaf@crsa.bu.edu; baoxin@crsa.bu.edu; lix@crsa.bu.edu; alan@crsa.bu.edu; wanner@crsa.bu.edu)

P. Lewis, Remote Sensing Unit, University College London, 26 Bedford Way,London, WC1H 0AP, UK. (e-mail: plewis@geog.ucl.ac.uk)

Jan-Peter Muller, Department of Photogrammetry and Surveying, University College London, Gower Street, London, WC1E 6BT, UK. (e-mail: jpmuller@ps.ucl.ac.uk)

Mike Bamsley, Department of Geography, University of Wales Swansea, Singleton Park, Swansea SA2 8PP, UK. (e-mail: m.barnsley@swansea.ac.uk)

(Received January 11, 1996; revised August 28, 1996; accepted October 9, 1996) 